\title{
Matrix coefficients of cohomologically induced representations
}

\author{
Binyong Sun
}

\begin{abstract}
We define integral formulas which produce certain matrix coefficients of cohomologically induced representations of real reductive groups. They are analogous to Harish-Chandra's Eisenstein integrals for matrix coefficients of ordinary induced representations, and generalize Flensted-Jensen's fundamental functions for discrete series.
\end{abstract}

\section{Introduction}

Let $G$ be a connected real Lie group such that:

(i) the Lie algebra $\mathfrak{g}_{0}$ of $G$ is reductive;

(ii) the connected Lie subgroup of $G$ with Lie algebra $\left[\mathfrak{g}_{0}, \mathfrak{g}_{0}\right]$ has a finite center.

Such a $G$ is called a connected real reductive group. Fix a maximal compact subgroup $K$ of $G$, which is connected and unique up to conjugation.

An elementary spherical function is a smooth function $\phi$ on $G / K$ with the following three properties:

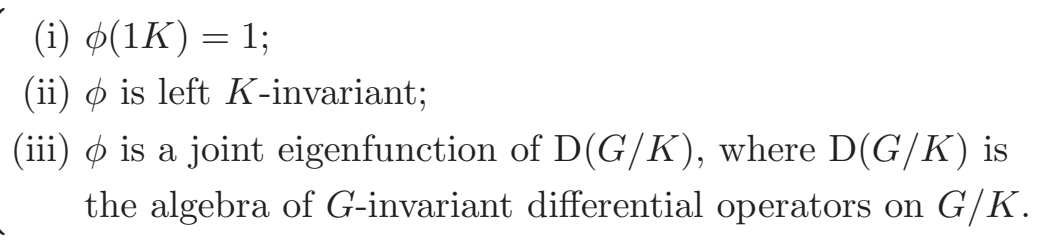

By using the Iwasawa decomposition $G=N A K$, Harish-Chandra found in [Har58] a pretty integral formula

$$
\phi_{\lambda}(x)=\int_{K} e^{\langle\lambda+\rho, H(k x)\rangle} d k, \quad x \in G,
$$

which produces elementary spherical functions on $G$, where $H$ is the map from $G$ onto $\mathfrak{a}_{0}=\operatorname{Lie}(A)$ defined by

$$
H(n \exp (a) k)=a, \quad n \in N, a \in \mathfrak{a}_{0}, k \in K,
$$

and $\rho \in \mathfrak{a}_{0}^{*}$ is half of the sum of positive roots (the positive system is the one that corresponds to $N)$. Moreover, he proved that a function on $G$ is elementary spherical if and only if it is of the form $\phi_{\lambda}$ for some $\lambda \in \mathfrak{a}_{0}^{*} \otimes_{\mathbb{R}} \mathbb{C}$. Note that a function on $G / K$ may be identified with a function on $G$ which is right $K$-invariant.

In terms of representation theory, elementary spherical functions can be expressed as matrix coefficients of irreducible spherical representations and its trivial $K$-type. In general, for an

Received 6 July 2005, accepted in final form 2 August 2006.

2000 Mathematics Subject Classification 22E46 (primary), 22E30 (secondary).

Keywords: matrix coefficient, cohomological induction, bottom layer, real reductive group.

This journal is (c) Foundation Compositio Mathematica 2007. 
admissible continuous representation $\pi$ of $G$ on a Hilbert space $V_{\pi}$, and a $K$-type $\alpha$, define

$$
\phi_{\pi, \alpha}(g)=p_{\alpha} \circ \pi(g) \circ j_{\alpha}, \quad g \in G,
$$

where $j_{\alpha}$ is the embedding of the $\alpha$-isotypic component $V_{\pi}(\alpha)$ into $V_{\pi}$, and $p_{\alpha}$ is the continuous linear projection from $V_{\pi}$ onto $V_{\pi}(\alpha)$ defined by

$$
p_{\alpha}(v)= \begin{cases}v, & \text { if } v \in V_{\pi}(\alpha), \\ 0, & \text { if } v \in V_{\pi}(\beta), \quad \text { for some } K \text {-type } \beta \neq \alpha .\end{cases}
$$

It is clear that $\phi_{\pi, \alpha}$ is a real analytic function on $G$ with values in $\operatorname{End}_{\mathbb{C}}\left(V_{\pi}(\alpha)\right)$. When $V_{\pi}(\alpha)$ has dimension 1, End $_{\mathbb{C}}\left(V_{\pi}(\alpha)\right)$ can be canonically identified with $\mathbb{C}$, and therefore $\phi_{\pi, \alpha}$ is actually a complex valued function. If $\pi$ is an irreducible spherical representation with the trivial $K$-type $\alpha_{0}$, then $V_{\pi}\left(\alpha_{0}\right)$ has dimension 1 , and the function $\phi_{\pi, \alpha_{0}}$ is an elementary spherical function. By the theory of spherical functions and spherical representations, all elementary spherical functions can be expressed as $\phi_{\pi, \alpha_{0}}$ for some irreducible spherical representation $\pi$.

In the sense of Vogan, $\alpha_{0}$ is the unique lowest $K$-type of every irreducible spherical representation. Therefore, it is interesting to study $\phi_{\pi, \alpha}$ for a general irreducible admissible representation $\pi$ and an arbitrary lowest $K$-type $\alpha$ of $\pi$. In particular, one may consider the following.

General Problem. Find an integral formula similar to (2) for $\phi_{\pi, \alpha}$, where $\pi$ is an arbitrary irreducible admissible representation, and $\alpha$ is an arbitrary lowest $K$-type of $\pi$.

Special cases of this General Problem have been extensively studied over the years. HarishChandra defined in [Har75] (or [Kna86, Wal92]) the Eisenstein integral which represents $\phi_{\pi, \alpha}$ when $\pi$ is a parabolically induced representation. In [Fle80a], Flensted-Jensen solved the problem when $\pi$ is a discrete series representation. $\mathrm{Li}$ and Wallach obtained a formula when $\pi$ is a lowest weight unitary representation [Li90]. Harris and Li gave formulas for some very special cases of cohomologically induced representations [HL98]. Some lower rank group cases have been explicitly calculated by mathematical physicists.

It is important to point out that Flensted-Jensen also attempted to give a solution of the General Problem [Fle80b]. His formulas produce functions with good properties. However, he has to consider the convergence problem since his formulas involve integrations over non-compact groups. In order to attack the convergence problem, he has to assume that the parameters in his formulas must be sufficiently regular.

The objective of this paper is to give an integral formula for $\phi_{\pi, \alpha}$ when $\pi$ is a cohomologically induced representation and $\alpha$ is in the bottom layer (we describe cohomological inductions and bottom layers in detail in $\S 5$ ). One of the good features of our formulas is that the integrations involved in our formulas are over compact groups. Hence, we do not have to deal with the convergence issue. In what follows, we explain precisely why our formulas are enough for a solution to the General Problem.

Let $\mathfrak{g}$ be the complexification of $\mathfrak{g}_{0}$. It is known that the function $\phi_{\pi, \alpha}$ depends only on $\alpha$ and the underlying $(\mathfrak{g}, K)$-module of $\pi$. Therefore, it makes sense to write $\phi_{M, \alpha}$ for a finitely generated admissible $(\mathfrak{g}, K)$-module $M$ (every $M$ has at least one globalization $\pi$ ).

Fix a Cartan involution $\theta$ on $G$ such that the fixed point group $G^{\theta}=K$. Recall that a parabolic subalgebra $\mathfrak{q}$ of $\mathfrak{g}$ is called a $\theta$-stable parabolic if

$$
\theta(\mathfrak{q})=\mathfrak{q}
$$

and

$\mathfrak{q} \cap \overline{\mathfrak{q}}$ is a Levi factor of $\mathfrak{q}$, 


\section{MATRIX COEFFicients}

where ${ }^{6-}$ is the complex conjugation relative to the real form $\mathfrak{g}_{0}$. Assume that $\alpha$ is a lowest $K$-type of an irreducible $(\mathfrak{g}, K)$-module $M$. It is automatic that $\alpha$ has multiplicity 1 in $M$. Vogan associated to $\alpha$ a $\theta$-stable parabolic $\mathfrak{q}$ (depending on some fixed positive system). Define

$$
G^{\prime}=\text { normalizer of } \mathfrak{q} \text { in } G \text {. }
$$

Then $G^{\prime}$ is a quasisplit connected real reductive group. Write

$$
\mathfrak{g}^{\prime}=\operatorname{Lie}\left(G^{\prime}\right) \otimes_{\mathbb{R}} \mathbb{C} \quad \text { and } \quad K^{\prime}=K \cap G^{\prime} .
$$

Let $\alpha^{\prime}$ be the $K^{\prime}$-type which is q-associated to $\alpha$ (Definition 3.1). Then $\alpha^{\prime}$ is fine with respect to $G^{\prime}$ in the sense of Vogan. By [SV98, Theorem 2.9], or [Vog81, Theorems 6.5.10 and 6.5.12], there is a $\left(\mathfrak{g}^{\prime}, K^{\prime}\right)$-module $P^{\prime}$ in the non-unitary principal series such that:

(i) $\alpha^{\prime}$ occurs in $P^{\prime}$ (automatically has multiplicity 1 );

(ii) $\alpha$ is in the bottom layer of $X_{G}$, occurring with multiplicity 1 , where $X_{G}=\mathcal{R}_{\mathfrak{q}}^{S}\left(P^{\prime}\right)$ is a cohomologically induced standard $(\mathfrak{g}, K)$-module (this is automatic from condition $(\mathrm{i})$, see the comments after Definition 5.4);

(iii) $M$ is isomorphic to the unique irreducible subquotient of $X_{G}$ containing the $K$-type $\alpha$.

From the third condition above we know that $\phi_{M, \alpha}$ can be canonically identified with $\phi_{X_{G}, \alpha}$. It is also known that $\phi_{P^{\prime}, \alpha^{\prime}}$ can be expressed in terms of the parameters of principal series by HarishChandra's Eisenstein integral. Therefore, if we can express $\phi_{X_{G}, \alpha}$ in terms of $\phi_{P^{\prime}, \alpha^{\prime}}$, we give a solution to the general problem.

In this paper, we define an integral (Definition 3.2) which is analogous to Harish-Chandra's Eisenstein integral and does express $\phi_{X_{G}, \alpha}$ in terms of $\phi_{P^{\prime}, \alpha^{\prime}}$ (Theorem 6.3). It generalizes FlensedJensen's fundamental functions for discrete series [Fle80a]. The crucial point is that we use the Langlands decomposition which is more general than the Iwasawa decomposition which was used by Flensted-Jensen [Fle80b].

A direct application of the formulas is to show certain positivity properties which are essential in the study of discrete spectra of theta correspondences [HL98, Li90, Li97]. For example, we find that $\phi_{\pi, \alpha}(x)$ is a positive definite operator when $\pi$ is an irreducible unitary representation with non-zero cohomology, $\alpha$ is the unique lowest $K$-type, and $x \in \exp \left(\mathfrak{p}_{0}\right)$, where $\mathfrak{p}_{0} \subset \mathfrak{g}_{0}$ is the -1 eigenspace of $\theta$.

In the next section, we recall Flensted-Jensen duality which relates functions on $G$ to functions on a Riemannian symmetric space. In $\S 3$, we introduce the integral and prove that the integral behaves well under the translations of $K$. In $\S 4$, we prove that the integral behaves well under the actions of $\mathrm{U}(\mathfrak{g})^{K}$. In $\S 5$, we recall cohomological inductions and bottom layers, and study the actions of $\mathrm{U}(\mathfrak{g})^{K}$ on bottom layers. The results of $\S \S 3,4$, and 5 imply that both our integral and $\phi_{M, \alpha}$ have three properties which are similar to those of (1), where $M$ is a cohomologically induced module and $\alpha$ is in the bottom layer. In $\S 6$, we use the uniqueness of functions with these properties to get the equality between our integral and $\phi_{M, \alpha}$, and give two interesting examples.

\section{Flensted-Jensen duality}

\subsection{Complexifications of groups}

We denote by $H_{\mathbb{C}}$ the universal complexification of a Lie group $H$. Let $u_{H}: H \rightarrow H_{\mathbb{C}}$ be the canonical homomorphism, and ${ }^{-}: H_{\mathbb{C}} \rightarrow H_{\mathbb{C}}$ be the anti-holomorphic automorphism on $H_{\mathbb{C}}$ which is identity on $u_{H}(H)$. Recall that the universal complexification map $u_{H}$ is defined by the following property [Hoc66]: if $H^{\prime}$ is a complex Lie group and $\phi: H \rightarrow H^{\prime}$ is a Lie group homomorphism, then there is a unique holomorphic homomorphism $\phi^{\prime}: H_{\mathbb{C}} \rightarrow H^{\prime}$ such that 
the following diagram commutes.

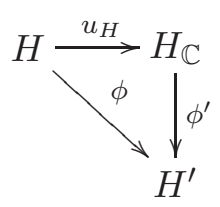

When $A$ is a connected real analytic manifold, $B$ is a connected complex manifold, and $c: A \rightarrow B$ is a real analytic map, we call $c$ a complexification map if its differential is a complexification of a real vector space everywhere. The following are well known:

(i) every Lie group has a unique universal complexification;

(ii) the universal complexification of a connected Lie group is connected;

(iii) if $H$ is a connected real reductive group, then $u_{H}$ is a complexification map and has a finite kernel;

(iv) if $H$ is compact, then $u_{H}$ is injective and $H_{\mathbb{C}}$ is a reductive complex group.

\subsection{Flensted-Jensen duality for groups}

Recall from $\S 1$ that $\theta$ is a Cartan involution on $G$ such that $K$ is its fixed point group. The involution $\theta$ induces a Lie algebra involution $\theta: \mathfrak{g} \rightarrow \mathfrak{g}$. Let

$$
\mathfrak{g}_{0}=\mathfrak{k}_{0}+\mathfrak{p}_{0} \quad \text { and } \quad \mathfrak{g}=\mathfrak{k}+\mathfrak{p}
$$

be the usual decompositions corresponding to $\theta$. Write

$$
\bar{\theta}=\theta \circ^{-}={ }^{-} \circ \theta: \mathfrak{g} \rightarrow \mathfrak{g} .
$$

The involution $\theta: G \rightarrow G$ also induces a holomorphic involution $\theta: G_{\mathbb{C}} \rightarrow G_{\mathbb{C}}$. Still write

$$
\bar{\theta}=\theta \circ^{-}={ }^{-} \circ \theta: G_{\mathbb{C}} \rightarrow G_{\mathbb{C}} .
$$

Let $U$ be the fixed points of $\bar{\theta}$ in $G_{\mathbb{C}}$. Note that $U$ is connected, and it is a maximal compact subgroup of $G_{\mathbb{C}}$ when $G$ has a compact center. We have a commutative diagram

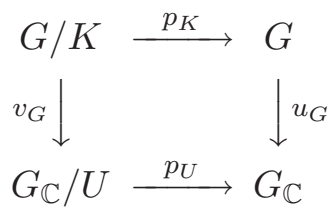

where $v_{G}$ is the map induced by $u_{G}, p_{K}$ is defined by

$$
p_{K}(x K)=x \theta\left(x^{-1}\right), \quad x \in G,
$$

and $p_{U}$ is defined by

$$
p_{U}(x U)=x \bar{\theta}\left(x^{-1}\right), \quad x \in G_{\mathbb{C}} .
$$

Note that both $u_{G}$ and $p_{U}$ are complexification maps.

Fix a finite-dimensional continuous representation $\tau$ of $K \times K$ on $\mathbf{V}$, and still denote by $\tau$ the holomorphic extension of the representation to $K_{\mathbb{C}} \times K_{\mathbb{C}}$. We have a smooth representation $T$ of $G \times G$ on $\mathrm{C}^{\infty}(G ; \mathbf{V})$ defined by

$$
\left(T_{g, h} \phi\right)(x)=\phi\left(g^{-1} x h\right)
$$

for all $g, h, x \in G, \phi \in \mathrm{C}^{\infty}(G ; \mathbf{V})$. By taking its differential, we get a representation $T$ of $\mathrm{U}(\mathfrak{g}) \otimes \mathrm{U}(\mathfrak{g})$ on $\mathrm{C}^{\infty}(G ; \mathbf{V})$. We also have a smooth representation $T$ of $G_{\mathbb{C}}$ on $\mathrm{C}^{\infty}\left(G_{\mathbb{C}} / U ; \mathbf{V}\right)$ by

$$
\left(T_{g} \psi\right)(x)=\psi\left(g^{-1} x\right)
$$




\section{MATRIX COEFFICIENTS}

for all $g \in G_{\mathbb{C}}, x \in G_{\mathbb{C}} / U, \psi \in \mathrm{C}^{\infty}\left(G_{\mathbb{C}} / U ; \mathbf{V}\right)$. Again by taking its differential and using the complexification

$$
\begin{array}{ccc}
\mathfrak{g} & \rightarrow & \mathfrak{g} \times \mathfrak{g}, \\
X & \mapsto & (X, \bar{\theta}(X)),
\end{array}
$$

we get a representation $T$ of $\mathrm{U}(\mathfrak{g}) \otimes \mathrm{U}(\mathfrak{g})$ on $\mathrm{C}^{\infty}\left(G_{\mathbb{C}} / U ; \mathbf{V}\right)$.

Define three spaces of $\tau$-spherical functions by

$$
\begin{gathered}
\mathrm{C}^{\infty}(G ; \tau)=\left\{\phi \in \mathrm{C}^{\infty}(G ; \mathbf{V}) \mid \phi\left(k_{1} x k_{2}^{-1}\right)=\tau\left(k_{1}, k_{2}\right) \phi(x), k_{1}, k_{2} \in K, x \in G\right\} ; \\
\mathrm{C}^{\infty}\left(G_{\mathbb{C}} / U ; \tau\right)=\left\{\phi \in \mathrm{C}^{\infty}\left(G_{\mathbb{C}} / U ; \mathbf{V}\right) \mid \phi(k x)=\tau(k, \bar{k}) \phi(x), k \in K_{\mathbb{C}}, x \in G_{\mathbb{C}} / U\right\} ; \\
\mathrm{C}^{\infty}(G / K ; \tau)=\left\{\phi \in \mathrm{C}^{\infty}(G / K ; \mathbf{V}) \mid \phi(k x)=\tau(k, k) \phi(x), k \in K, x \in G / K\right\} .
\end{gathered}
$$

The spaces $\mathrm{C}^{\omega}(G ; \tau), \mathrm{C}^{\omega}\left(G_{\mathbb{C}} / U ; \tau\right)$ and $\mathrm{C}^{\omega}(G / K ; \tau)$ are defined similarly, here the superscript ' $\omega$ ' stands for real analytic functions. Note that both $\mathrm{C}^{\omega}(G ; \tau)$ and $\mathrm{C}^{\omega}\left(G_{\mathbb{C}} / U ; \tau\right)$ are stable under $T_{\mathrm{U}(\mathfrak{g})^{K} \otimes \mathrm{U}(\mathfrak{g})^{K}}$, where $\mathrm{U}(\mathfrak{g})^{K}$ is as usual the $K$-fixed vectors in $\mathrm{U}(\mathfrak{g})$ under the adjoint action.

The following is essentially [Fle80a, Theorem 7.1]. We omit its proof.

THEOREM 2.1. We have the following.

(i) The pull backs of $p_{K}$ and $v_{G}$ in the diagram (3) induce canonical isomorphisms of vector spaces:

$$
\mathrm{C}^{\infty}(G ; \tau) \cong \mathrm{C}^{\infty}(G / K ; \tau) \cong \mathrm{C}^{\infty}\left(G_{\mathbb{C}} / U ; \tau\right) .
$$

(ii) The above identifications induce linear isomorphisms

$$
\mathrm{C}^{\omega}(G ; \tau) \cong \mathrm{C}^{\omega}(G / K ; \tau) \cong \mathrm{C}^{\omega}\left(G_{\mathbb{C}} / U ; \tau\right) .
$$

(iii) The identification

$$
\mathrm{C}^{\omega}(G ; \tau) \cong \mathrm{C}^{\omega}\left(G_{\mathbb{C}} / U ; \tau\right)
$$

respects the actions of $\mathrm{U}(\mathfrak{g})^{K} \otimes \mathrm{U}(\mathfrak{g})^{K}$.

\section{The integral}

\subsection{The definition}

As in $\S 1, \mathfrak{q}$ is a $\theta$-stable parabolic, $G^{\prime}$ is the normalizer of $\mathfrak{q}$ in $G$,

$$
\mathfrak{g}^{\prime}=\operatorname{Lie}\left(G^{\prime}\right) \otimes_{\mathbb{R}} \mathbb{C} \quad \text { and } \quad K^{\prime}=K \cap G^{\prime} .
$$

It is known that $G^{\prime}$ is a connected real reductive group and $K^{\prime}$ is a maximal compact subgroup of it. The involution $\theta$ stabilizes $G^{\prime}$ and induces a holomorphic involution of $G_{\mathbb{C}}^{\prime}$, which is also denoted by $\theta$. Let

$$
\bar{\theta}=\theta \circ^{-}={ }^{-} \circ \theta
$$

as before. Denote by $U^{\prime}$ the fixed points of $\bar{\theta}$ in $G_{\mathbb{C}}^{\prime}$. Let $\mathfrak{n}$ be the nilpotent radical of $\mathfrak{q} \cap[\mathfrak{g}, \mathfrak{g}]$. Then

$$
\mathfrak{q}=\mathfrak{g}^{\prime} \oplus \mathfrak{n}
$$

Denote by $N$ the connected complex subgroup of $G_{\mathbb{C}}$ with Lie algebra $\mathfrak{n}$. Denote by

$$
j_{\mathbb{C}}: G_{\mathbb{C}}^{\prime} \rightarrow G_{\mathbb{C}}
$$

the complexification of the embedding $j: G^{\prime} \rightarrow G$. The Langlands decomposition

$$
G_{\mathbb{C}}=N j_{\mathbb{C}}\left(G_{\mathbb{C}}^{\prime}\right) U
$$


enables us to define a real analytic map

$$
\begin{aligned}
H_{\mathfrak{q}}: \quad G_{\mathbb{C}} / U & \rightarrow G_{\mathbb{C}}^{\prime} / U^{\prime} \\
n j_{\mathbb{C}}(g) U & \mapsto g U^{\prime}
\end{aligned}
$$

for all $n \in N$ and $g \in G_{\mathbb{C}}^{\prime}$.

The following conventions will be used freely: if $H$ is a Lie group, $\mathfrak{c}$ is a complex subspace of a complex Lie algebra, and $H$ acts on $\mathfrak{c}$ by certain adjoint action, then $\delta_{\mathfrak{c}}$ is the non-unitary character on $H$ defined by

$$
\delta_{\mathfrak{c}}(x)=\operatorname{Det}\left(\left.\operatorname{Ad}(x)\right|_{\mathfrak{c}}\right)
$$

If $\sigma$ is a continuous finite-dimensional representation of a Lie group $H$, then its differential and its holomorphic extension to $H_{\mathbb{C}}$ are still denoted by $\sigma$. Note that

$$
\delta_{\mathfrak{c}}(X)=\operatorname{Tr}\left(\left.\operatorname{ad}(X)\right|_{\mathfrak{c}}\right), \quad X \in \operatorname{Lie}(H) \otimes_{\mathbb{R}} \mathbb{C} .
$$

Define

$$
\delta_{\mathfrak{n}}^{+}\left(x U^{\prime}\right)=\delta_{\mathfrak{n}}\left(x \bar{\theta}\left(x^{-1}\right)\right), \quad x U^{\prime} \in G_{\mathbb{C}}^{\prime} / U^{\prime} .
$$

It is not difficult to see that $\delta_{\mathfrak{n}}^{+}$has positive values everywhere. However, this fact plays no roll in this paper.

Definition 3.1. (i) If $\sigma$ is a finite-dimensional continuous representation of $K$ on $W$, define a representation $\sigma^{\prime}$ of $K^{\prime}$ on

$$
W^{\prime}=\{v \in W \mid \sigma(\mathfrak{n} \cap \mathfrak{k}) v=0\}
$$

by

$$
\sigma^{\prime}(k) v=\delta_{\overline{\mathfrak{n}} \cap \mathfrak{p}}(k) \sigma(k) v, \quad k \in K^{\prime}, v \in W^{\prime} .
$$

Then $\sigma^{\prime}$ is called the representation $\mathfrak{q}$-associated to $\sigma$.

(ii) If $\tau$ is a finite-dimensional continuous representation of $K \times K$ on $\mathbf{V}$, define a representation $\tau^{\prime}$ of $K^{\prime} \times K^{\prime}$ on

$$
\mathbf{V}^{\prime}=\{v \in \mathbf{V} \mid \tau(X, Y) v=0 \text { for all } X \in \mathfrak{n} \cap \mathfrak{k}, Y \in \overline{\mathfrak{n}} \cap \mathfrak{k}\}
$$

by

$$
\tau^{\prime}(k, l) v=\delta_{\overline{\mathfrak{n}} \cap \mathfrak{p}}(k) \delta_{\mathfrak{n} \cap \mathfrak{p}}(l) \tau(k, l) v, \quad k, l \in K^{\prime}, v \in \mathbf{V}^{\prime} .
$$

Then $\tau^{\prime}$ is called the representation $\mathfrak{q} \times \overline{\mathfrak{q}}$-associated to $\tau$.

Now let $\tau^{\prime}$ be the representation $\mathfrak{q} \times \overline{\mathfrak{q}}$-associated to $\tau$ as in the second part of the definition. We define $\mathrm{C}^{\infty}\left(G_{\mathbb{C}}^{\prime} / U^{\prime} ; \tau^{\prime}\right)$ in the same way as that of $\mathrm{C}^{\infty}\left(G_{\mathbb{C}} / U ; \tau\right)$ in $(5)$. We also use similar notation for other spaces. Now we have enough preparations to define our integral.

Definition 3.2. For any

$$
\phi \in \mathrm{C}^{\infty}\left(G_{\mathbb{C}}^{\prime} / U^{\prime} ; \tau^{\prime}\right)
$$

define the integral

$$
E_{\mathfrak{q}}(\phi: x)=\int_{K} \tau\left(k^{-1}, k^{-1}\right)\left(\delta_{\mathfrak{n}}^{+} \phi\right)\left(H_{\mathfrak{q}}(k x)\right) d k, \quad x \in G_{\mathbb{C}} / U .
$$

As usual, we use normalized Haar measures on compact groups for integration. Our integral is very similar to Harish-Chandra's Eisenstein integral for a real parabolic subgroup. The remainder of this section is used to establish one of the main results in this paper.

Theorem 3.3. We have $E_{\mathfrak{q}}(\phi) \in \mathrm{C}^{\infty}\left(G_{\mathbb{C}} / U ; \tau\right)$ for all $\phi \in \mathrm{C}^{\infty}\left(G_{\mathbb{C}}^{\prime} / U^{\prime} ; \tau^{\prime}\right)$. 


\section{MATRIX COEFFICIENTS}

\subsection{An integral formula}

In this subsection, we establish an integral formula which is crucial for the proof of Theorem 3.3. The formula is a stronger version of [KV95, Corollary 11.40]. We formulate it in full generality, although we only need to apply it to the complex group $K_{\mathbb{C}}$, since the proof of the general case in no more difficult than that of the special case. The notation of this subsection is not used in other parts of this paper.

Let $G$ be a real reductive Lie group (which the reader may assume to be connected), and $\theta$ be a Cartan involution on $G$. Let $\mathfrak{q}_{0}$ be a parabolic subalgebra of $\mathfrak{g}_{0}=\operatorname{Lie}(G)$. We introduce the following notation:

- $K$ is the maximal compact subgroup of $G$ corresponding to $\theta$;

- $\mathfrak{g}_{0}=\mathfrak{k}_{0}+\mathfrak{p}_{0}$ is the Cartan decomposition corresponding to $\theta$;

- $Q$ is the normalizer of $\mathfrak{q}_{0}$ in $G$;

- $L=Q \cap \theta(Q)$;

- $Q=L N$ is the Levi decomposition;

- $\mathfrak{q}_{0}=\mathfrak{l}_{0}+\mathfrak{n}_{0}$ is the corresponding Levi decomposition at the Lie algebra level;

- $\mathfrak{l}_{0}^{\prime}=\mathfrak{l}_{0} \cap \mathfrak{p}_{0}$;

- $\delta: L \rightarrow \mathbb{R}^{\times}$is the homomorphism defined by the absolute value of the determinant of the adjoint representation of $L$ on $\mathfrak{n}_{0}$.

By Langlands decomposition, the map

$$
\begin{aligned}
N \times \mathfrak{l}_{0}^{\prime} \times K & \rightarrow G, \\
(n, X, k) & \mapsto n \exp (X) k
\end{aligned}
$$

is a real analytic diffeomorphism. We define two maps $\kappa: G \rightarrow K$ and $I: G \rightarrow L$ by

$$
\kappa(n a k)=k, \quad I(n a k)=a
$$

for all $n \in N, a \in \exp \left(\mathfrak{l}_{0}^{\prime}\right), k \in K$. If $a \in G$, we define a map $\kappa^{a}: K \rightarrow K$ by

$$
\kappa^{a}(k)=\kappa(k a) .
$$

Proposition 3.4. If $f$ is a continuous function on $K, a \in G$, then

$$
\int_{K} f\left(\kappa^{a}(k)\right) d k=\int_{K} f(k) \delta\left(I\left(k a^{-1}\right)\right) d k .
$$

The proof of Proposition 3.4 follows from Lemma 3.5-3.8 given in the remainder of this subsection.

Lemma 3.5. The maps $\kappa^{a}$ and $\kappa^{a^{-1}}$ are inverse to each other. Hence, $\kappa^{a}$ is an analytic diffeomorphism.

Proof. Let $k \in K$ and $k a=n_{1} s_{1} k_{1}$, where $n_{1} \in N, s_{1} \in \exp \left(\mathfrak{l}_{0}^{\prime}\right)$, and $k_{1} \in K$. Then we have

$$
k_{1} a^{-1}=s_{1}^{-1} n_{1}^{-1} k=\left(s_{1}^{-1} n_{1}^{-1} s_{1}\right) s_{1}^{-1} k .
$$

Hence,

Change $a$ to $a^{-1}$, we get $\kappa^{a} \kappa^{a^{-1}}(k)=k$.

$$
\kappa^{a^{-1}} \kappa^{a}(k)=\kappa^{a^{-1}}\left(k_{1}\right)=\kappa\left(k_{1} a^{-1}\right)=k .
$$

If $a \in G$, we denote by $T_{a}$ the right translation of $a$ on $Q \backslash G$. We give $Q \backslash G$ the unique $K$-invariant measure with total mass 1 . 
Lemma 3.6. If $k \in K$, then the Jacobian of $T_{a}$ at $Q k$ is

$$
J_{T_{a}}(Q k)=\delta(I(k a))
$$

This should be known. We give a proof for the sake of completeness.

Proof. Let $k a=n s \kappa(k a)$, where $n \in N$ and $s=I(k a)$. We have the following commutative diagram.

$$
\begin{array}{cc}
Q \backslash G \stackrel{T_{a}}{\longrightarrow} Q \backslash G \\
T_{k^{-1}} \downarrow \\
Q \backslash G \stackrel{T_{n s}}{\longrightarrow} Q \backslash G
\end{array}
$$

Since $T_{k^{-1}}$ and $T_{\kappa(k a)^{-1}}$ preserve the measure,

$$
J_{T_{a}}(Q k)=J_{T_{n s}}(Q 1) .
$$

The right-hand side is the absolute value of the determinant of the tangent map $\left.\mathrm{d} T_{n s}\right|_{Q}$ at the point $Q$. We have another commutative diagram.

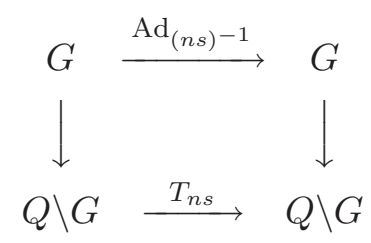

By taking the tangent map at the identity, we get the following diagram.

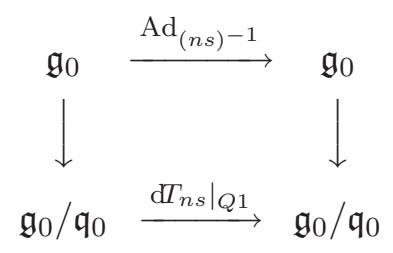

Hence,

$$
\begin{aligned}
J_{T_{a}}(Q k) & =J_{T_{n s}}(Q 1)=\left|\operatorname{Det}\left(\left.\mathrm{d} T_{n s}\right|_{Q 1}\right)\right| \\
& =\frac{\left|\operatorname{Det}\left(\operatorname{Ad}_{(n s)^{-1}}\right)\right|}{\left|\operatorname{Det}\left(\left.\operatorname{Ad}_{(n s)^{-1}}\right|_{\mathfrak{q}_{0}}\right)\right|}=\frac{1}{\delta\left(s^{-1}\right)}=\delta(s) .
\end{aligned}
$$

For any $a \in G$, let $J_{a}$ be the Jacobian of $\kappa^{a}$.

Lemma 3.7. The Jacobian $J_{a}$ is left $K \cap Q$ invariant.

Proof. For any $k \in K$, we use $L_{k}$ to denote the left translation on $K$ by $k$. The lemma comes from the fact that if $k \in K \cap Q$, then the following diagram commutes.

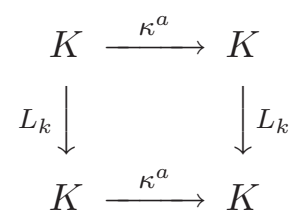

We omit the easy proof of this fact.

Lemma 3.8. We have $J_{a}(k)=\delta(I(k a))$ for all $k \in K$. 


\section{MATRIX COEFFICIENTS}

Proof. Let $\pi$ be the map $K \rightarrow Q \backslash G, k \mapsto Q k$. By the above lemma, there is a function $J_{a}^{\prime}$ on $Q \backslash G$ such that $J_{a}^{\prime} \circ \pi=J_{a}$. For all continuous function $f$ on $Q \backslash G$, we have

$$
\begin{aligned}
\int_{Q \backslash G} f\left(T_{a}(x)\right) J_{a}^{\prime}(x) d x & =\int_{K} f\left(T_{a}(\pi(k))\right) J_{a}^{\prime}(\pi(k)) d k \\
& =\int_{K} f\left(\left(\pi\left(\kappa^{a}(k)\right)\right)\right) J_{a}(k) d k \quad\left(T_{a} \circ \pi=\pi \circ \kappa^{a}\right) \\
& =\int_{K} f(\pi(k)) d k \\
& =\int_{Q \backslash G} f(x) d x \\
& =\int_{Q \backslash G} f\left(T_{a}(x)\right) J_{T_{a}}(x) d x .
\end{aligned}
$$

Hence, $J_{a}^{\prime}=J_{T_{a}}$. We conclude the proof by Lemma 3.6.

Proof of Proposition 3.4. We have

$$
\begin{aligned}
\int_{K} f\left(\kappa^{a}(k)\right) d k & =\int_{K} f\left(\kappa^{a}\left(\kappa^{a^{-1}}(k)\right)\right) J_{a^{-1}}(k) d k \\
& =\int_{K} f(k) \delta\left(I\left(k a^{-1}\right)\right) d k
\end{aligned}
$$

\subsection{A proof of Theorem 3.3}

We now return to the notation of $\S 3.1$. We apply the results obtained in the last subsection to the group $K_{\mathbb{C}}$. Let $\mathfrak{k}_{0}^{\prime}$ be the Lie algebra of $K^{\prime}$. Let $N_{c}$ be the connected subgroup of $K_{\mathbb{C}}$ with Lie algebra $\mathfrak{n} \cap \mathfrak{k}$. Now the parabolic subgroup is $N_{c} K_{\mathbb{C}}^{\prime}$. The map (15) in this case is

$$
\begin{aligned}
N_{c} \times \sqrt{-1} \mathfrak{k}_{0}^{\prime} \times K & \rightarrow K_{\mathbb{C}}, \\
(n, X, k) & \mapsto n \exp (X) k .
\end{aligned}
$$

Define $\kappa: K_{\mathbb{C}} \rightarrow K, I: K_{\mathbb{C}} \rightarrow K_{\mathbb{C}}^{\prime}, \kappa^{a}: K \rightarrow K, a \in K_{\mathbb{C}}$, and $\delta: K_{\mathbb{C}}^{\prime} \rightarrow \mathbb{R}^{\times}$as in the last subsection.

LEMma 3.9. We have

$$
H_{\mathfrak{q}}(k a x)=\left(I\left(\kappa^{a}(k) a^{-1}\right)\right)^{-1} H_{\mathfrak{q}}\left(\kappa^{a}(k) x\right)
$$

for all $k \in K, a \in K_{\mathbb{C}}$ and $x \in G_{\mathbb{C}} / U$.

Proof. Write $k a=n^{\prime} a^{\prime} k^{\prime}$, where $n^{\prime} \in N_{c}, a^{\prime} \in \exp \left(\sqrt{-1} k_{0}^{\prime}\right), k^{\prime} \in K^{\prime}$. Then

$$
\kappa^{a}(k) a^{-1}=k^{\prime} a^{-1}=a^{\prime-1} n^{\prime-1} k=\left(a^{\prime-1} n^{\prime-1} a^{\prime}\right) a^{\prime-1} k .
$$

Therefore,

$$
I\left(\kappa^{a}(k) a^{-1}\right)=a^{\prime-1} .
$$

As $H_{\mathfrak{q}}$ is $G_{\mathbb{C}^{-}}^{\prime}$-equivariant and $N$-invariant, we have

$$
\begin{aligned}
\left(I\left(\kappa^{a}(k) a^{-1}\right)\right)^{-1} H_{\mathfrak{q}}\left(\kappa^{a}(k) x\right) & =a^{\prime} H_{\mathfrak{q}}\left(\kappa^{a}(k) x\right)=H_{\mathfrak{q}}\left(a^{\prime} \kappa^{a}(k) x\right) \\
& =H_{\mathfrak{q}}\left(a^{\prime} k^{\prime} x\right)=H_{\mathfrak{q}}\left(n^{\prime-1} k a x\right)=H_{\mathfrak{q}}(k a x) .
\end{aligned}
$$

Define a representation $\left.\tau\right|_{K_{\mathbb{C}}}$ of $K_{\mathbb{C}}$ on $\mathbf{V}$ by

$$
\left.\tau\right|_{K_{\mathbb{C}}}(k)=\tau(k, \bar{k}),
$$


and a representation $\left.\tau\right|_{K}$ of $K$ on $\mathbf{V}$ by

$$
\left.\tau\right|_{K}(k)=\tau(k, k)
$$

LEMma 3.10. We have

$$
\left(\delta_{\mathfrak{n}}^{+} \phi\right)(k x)=\left.\delta_{\mathfrak{n} \cap \mathfrak{k}}(k) \delta_{\overline{\mathfrak{n}} \cap \mathfrak{k}}(\bar{k}) \tau\right|_{K_{\mathbb{C}}}(k)\left(\delta_{\mathfrak{n}}^{+} \phi\right)(x)
$$

for all $k \in K_{\mathbb{C}}^{\prime}, x \in G_{\mathbb{C}}^{\prime} / U^{\prime}$, and $\phi \in \mathrm{C}^{\infty}\left(G_{\mathbb{C}}^{\prime} / U^{\prime} ; \tau^{\prime}\right)$.

Proof.

$$
\begin{aligned}
\delta_{\mathfrak{n}}^{+} \phi(k x) & =\delta_{\mathfrak{n}}^{+}(k x) \phi(k x) \\
& =\delta_{\mathfrak{n}}(k) \delta_{\mathfrak{n}}\left(\bar{k}^{-1}\right) \delta_{\mathfrak{n}}^{+}(x) \tau^{\prime}(k, \bar{k}) \phi(x) \quad \text { by }(9) \\
& =\delta_{\mathfrak{n}}(k) \delta_{\mathfrak{n}}\left(\bar{k}^{-1}\right) \delta_{\overline{\mathfrak{n}} \cap \mathfrak{p}}(k) \delta_{\mathfrak{n} \cap \mathfrak{p}}(\bar{k}) \delta_{\mathfrak{n}}^{+}(x) \tau(k, \bar{k}) \phi(x) \quad \text { by }(13) \\
& =\left.\delta_{\mathfrak{n} \cap \mathfrak{k}}(k) \delta_{\overline{\mathfrak{n}} \cap \mathfrak{k}}(\bar{k}) \tau\right|_{K_{\mathbb{C}}}(k)\left(\delta_{\mathfrak{n}}^{+} \phi\right)(x) .
\end{aligned}
$$

We omit the proof of the following elementary lemma.

Lemma 3.11. We have

for all $k \in K_{\mathbb{C}}^{\prime}$.

$$
\delta\left(k^{-1}\right) \delta_{\mathfrak{n} \cap \mathfrak{k}}(k) \delta_{\overline{\mathfrak{n}} \cap \mathfrak{k}}(\bar{k})=1
$$

Proof of Theorem 3.3. Let $a \in K_{\mathbb{C}}$ and $x \in G_{\mathbb{C}} / U$. Write $k^{\prime}=\kappa^{a}(k)$. Then Lemma 3.9 implies

$$
\begin{aligned}
E_{\mathfrak{q}}(\phi: a x) & =\left.\int_{K} \tau\right|_{K}\left(k^{-1}\right)\left(\delta_{\mathfrak{n}}^{+} \phi\right)\left(H_{\mathfrak{q}}(k a x)\right) d k \\
& =\left.\int_{K} \tau\right|_{K}\left(\left(\kappa^{a^{-1}}\left(k^{\prime}\right)\right)^{-1}\right)\left(\delta_{\mathfrak{n}}^{+} \phi\right)\left(\left(I\left(k^{\prime} a^{-1}\right)\right)^{-1}\left(H_{\mathfrak{q}}\left(k^{\prime} x\right)\right)\right) d k .
\end{aligned}
$$

Now by using Proposition 3.4, we have

$$
E_{\mathfrak{q}}(\phi: a x)=\left.\int_{K} \delta\left(I\left(k a^{-1}\right)\right) \tau\right|_{K}\left(\left(\kappa^{a^{-1}}(k)\right)^{-1}\right)\left(\delta_{\mathfrak{n}}^{+} \phi\right)\left(\left(I\left(k a^{-1}\right)\right)^{-1} H_{\mathfrak{q}}(k x)\right) d k .
$$

Write $I^{\prime}=\left(I\left(k a^{-1}\right)\right)^{-1}$ and $k^{\prime \prime}=\left(\kappa^{a^{-1}}(k)\right)^{-1}$ for simplicity. By Lemma 3.10, we have

$$
\begin{aligned}
E_{\mathfrak{q}}(\phi: a x) & =\left.\left.\int_{K} \delta\left(I^{\prime-1}\right) \tau\right|_{K}\left(k^{\prime \prime}\right) \delta_{\mathfrak{n} \cap \mathfrak{k}}\left(I^{\prime}\right) \delta_{\overline{\mathfrak{n}} \cap \mathfrak{k}}\left(\bar{I}^{\prime}\right) \tau\right|_{K_{\mathbb{C}}}\left(I^{\prime}\right)\left(\delta_{\mathfrak{n}}^{+} \phi\right)\left(H_{\mathfrak{q}}(k x)\right) d k \\
& =\left.\int_{K}\left(\delta\left(I^{\prime-1}\right) \delta_{\mathfrak{n} \cap \mathfrak{k}}\left(I^{\prime}\right) \delta_{\overline{\mathfrak{n}} \cap \mathfrak{k}}\left(\bar{I}^{\prime}\right)\right) \tau\right|_{K_{\mathbb{C}}}\left(k^{\prime \prime} I^{\prime}\right)\left(\delta_{\mathfrak{n}}^{+} \phi\right)\left(H_{\mathfrak{q}}(k x)\right) d k .
\end{aligned}
$$

Note that $k^{\prime \prime} I^{\prime}=a k^{-1} n$ for some $n \in N_{c}$, and $N_{c}$ fix the values of $\phi$ under the action $\left.\tau\right|_{K_{\mathbb{C}}}$. Therefore, Lemma 3.11 implies

$$
E_{\mathfrak{q}}(\phi: a x)=\left.\int_{K} \tau\right|_{K_{\mathbb{C}}}\left(a k^{-1}\right)\left(\delta_{\mathfrak{n}}^{+} \phi\right)\left(H_{\mathfrak{q}}(k x)\right) d k=\left.\tau\right|_{K_{\mathbb{C}}}(a) E_{\mathfrak{q}}(\phi: x) .
$$

\section{Differential equations satisfied by the integral}

\subsection{The differential equations}

We recall the generalized Harish-Chandra homomorphism

$$
\xi: \mathrm{U}(\mathfrak{g})^{K} \rightarrow \mathrm{U}\left(\mathfrak{g}^{\prime}\right)^{K^{\prime}}
$$

from $[\operatorname{Vog} 79, \S 3]$. 


\section{MATRIX COEFFICIENTS}

By the Poincaré-Birkhoff-Witt theorem,

$$
\mathrm{U}(\mathfrak{g})=\mathrm{U}\left(\mathfrak{g}^{\prime}\right) \oplus(\mathfrak{n} \mathrm{U}(\mathfrak{g})+\mathrm{U}(\mathfrak{g}) \overline{\mathfrak{n}}) .
$$

It is known that

$$
\mathrm{U}(\mathfrak{g})^{K} \subset \mathrm{U}\left(\mathfrak{g}^{\prime}\right)^{K^{\prime}} \oplus(\mathfrak{n} \mathrm{U}(\mathfrak{g}) \cap \mathrm{U}(\mathfrak{g}) \overline{\mathfrak{n}}) .
$$

Denote the projection to the first factor by

$$
\tilde{\xi}: \mathrm{U}(\mathfrak{g})^{K} \rightarrow \mathrm{U}\left(\mathfrak{g}^{\prime}\right)^{K^{\prime}} .
$$

It is also known that $\tilde{\xi}$ is an algebra homomorphism. Let

$$
\eta_{\mathfrak{q}}: \mathrm{U}\left(\mathfrak{g}^{\prime}\right) \rightarrow \mathrm{U}\left(\mathfrak{g}^{\prime}\right)
$$

be the algebra homomorphism such that

$$
\eta_{\mathfrak{q}}(X)=X+\delta_{\mathfrak{n}}(X)
$$

for all $X \in \mathfrak{g}^{\prime}$. Note that

$$
\delta_{\mathfrak{n}}(X)=\operatorname{Tr}\left(\left.\operatorname{ad}(X)\right|_{\mathfrak{n}}\right),
$$

which is twice the shift in the definition of Harish-Chandra homomorphism. It is easy to see that $\eta_{\mathfrak{q}}$ maps $\mathrm{U}\left(\mathfrak{g}^{\prime}\right)^{K^{\prime}}$ onto itself. The map $\xi$ is now defined by

$$
\xi=\eta_{\mathfrak{q}} \circ \tilde{\xi}: \mathrm{U}(\mathfrak{g})^{K} \rightarrow \mathrm{U}\left(\mathfrak{g}^{\prime}\right)^{K^{\prime}} .
$$

Recall that there is an anti-automorphism $\vee: \mathrm{U}(\mathfrak{g}) \rightarrow \mathrm{U}(\mathfrak{g})$ which maps $X$ to $-X$ for all $X \in \mathfrak{g}$. It preserves both $\mathrm{U}(\mathfrak{g})^{K}$ and $\mathrm{U}\left(\mathfrak{g}^{\prime}\right)^{K^{\prime}}$. If $A, B$ are two subalgebras of $\mathrm{U}(\mathfrak{g})$ which are stable under ${ }^{\vee}$, and if $\eta: A \rightarrow B$ is any algebra homomorphism, then denote by $\eta^{\vee}: A \rightarrow B$ the algebra homomorphism such that the diagram

commutes.

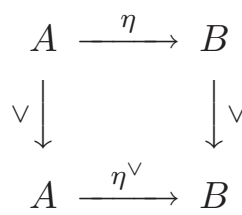

Let $\mathbf{V}, \tau, \mathbf{V}^{\prime}$ and $\tau^{\prime}$ be as in $\S 3.1$. Recall that we have an action $T$ of $\mathrm{U}(\mathfrak{g}) \otimes \mathrm{U}(\mathfrak{g})$ on $\mathrm{C}^{\infty}\left(G_{\mathbb{C}} / U ; \mathbf{V}\right)$ and $\mathrm{C}^{\infty}(G ; \mathbf{V})$. Denote by $T^{\prime}$ the analogous action of $\mathrm{U}\left(\mathfrak{g}^{\prime}\right) \otimes \mathrm{U}\left(\mathfrak{g}^{\prime}\right)$ on $\mathrm{C}^{\infty}\left(G_{\mathbb{C}}^{\prime} / U^{\prime} ; \mathbf{V}^{\prime}\right)$ and $\mathrm{C}^{\infty}\left(G^{\prime} ; \mathbf{V}^{\prime}\right)$. Recall that

$$
T_{\mathrm{U}(\mathfrak{g})^{K} \otimes \mathrm{U}(\mathfrak{g})^{K}} \quad \text { stabilizes } \mathrm{C}^{\infty}\left(G_{\mathbb{C}} / U ; \tau\right),
$$

and, similarly,

$$
T_{\mathrm{U}\left(\mathfrak{g}^{\prime}\right)^{K^{\prime}} \otimes \mathrm{U}\left(\mathfrak{g}^{\prime}\right)^{K^{\prime}}}^{\prime} \quad \text { stabilizes } \mathrm{C}^{\infty}\left(G_{\mathbb{C}}^{\prime} / U^{\prime} ; \tau^{\prime}\right)
$$

The main result of this section is the following theorem.

Theorem 4.1. For all $X \otimes Y \in \mathrm{U}(\mathfrak{g})^{K} \otimes \mathrm{U}(\mathfrak{g})^{K}$ and $\phi \in \mathrm{C}^{\infty}\left(G_{\mathbb{C}}^{\prime} / U^{\prime} ; \tau^{\prime}\right)$,

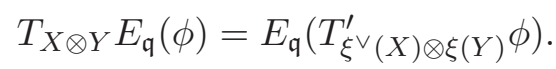

\subsection{Proof of Theorem 4.1}

Note that we also have

$$
\mathrm{U}(\mathfrak{g})^{K} \subset \mathrm{U}\left(\mathfrak{g}^{\prime}\right)^{K^{\prime}} \oplus(\overline{\mathfrak{n}} \mathrm{U}(\mathfrak{g}) \cap \mathrm{U}(\mathfrak{g}) \mathfrak{n}) .
$$

The projection to the first factor is just the algebra homomorphism

$$
\tilde{\xi}^{\vee}: \mathrm{U}(\mathfrak{g})^{K} \rightarrow \mathrm{U}\left(\mathfrak{g}^{\prime}\right)^{K^{\prime}}
$$




\section{B. SUN}

Lemma 4.2. Let $X \otimes Y \in \mathrm{U}(\mathfrak{g})^{K} \otimes \mathrm{U}(\mathfrak{g})^{K}$ and $f \in \mathrm{C}^{\infty}\left(G_{\mathbb{C}} / U ; \mathbf{V}\right)$. If $f$ is $N$-invariant, then

$$
T_{X \otimes Y} f=T_{\tilde{\xi}^{\vee}(X) \otimes \tilde{\xi}(Y)} f .
$$

Proof. Let $X=X^{\prime}+X^{\prime \prime}, Y=Y^{\prime}+Y^{\prime \prime}$, where $X^{\prime}=\tilde{\xi}^{\vee}(X), X^{\prime \prime} \in U(\mathfrak{g}) \mathfrak{n}, Y^{\prime}=\tilde{\xi}(Y), Y^{\prime \prime} \in U(\mathfrak{g}) \overline{\mathfrak{n}}$. Note that under the complexification

$$
\begin{aligned}
1 \times \bar{\theta}: & \mathfrak{g} \rightarrow \mathfrak{g} \times \mathfrak{g}, \\
v & \mapsto(v, \bar{\theta}(v)),
\end{aligned}
$$

the Lie algebra of $N$ has a complexification $\mathfrak{n} \times \overline{\mathfrak{n}}$. Therefore, the $N$ invariance of $f$ implies $T_{\mathfrak{n} \times \overline{\mathfrak{n}}} f=0$, i.e. $T_{\mathfrak{n} \otimes 1+1 \otimes \overline{\mathfrak{n}}} f=0$. So we have

$$
T_{X \otimes Y} f=T_{X^{\prime} \otimes Y^{\prime}} f=T_{\tilde{\xi}^{\vee}(X) \otimes \tilde{\xi}(Y)} f .
$$

Lemma 4.3. Let $X \otimes Y \in \mathrm{U}(\mathfrak{g})^{K} \otimes \mathrm{U}(\mathfrak{g})^{K}$ and $\phi \in \mathrm{C}^{\infty}\left(G_{\mathbb{C}}^{\prime} / U^{\prime} ; \mathbf{V}^{\prime}\right)$. Then

$$
T_{\tilde{\xi} \vee(X) \otimes \tilde{\xi}(Y)}^{\prime}\left(\delta_{\mathfrak{n}}^{+} \phi\right)=\delta_{\mathfrak{n}}^{+} T_{\xi^{\vee}(X) \otimes \xi(Y)}^{\prime}(\phi) .
$$

Proof. Let

$$
\eta_{\overline{\mathfrak{q}}}: \mathrm{U}\left(\mathfrak{g}^{\prime}\right) \rightarrow \mathrm{U}\left(\mathfrak{g}^{\prime}\right)
$$

be the algebra homomorphism such that $\eta_{\overline{\mathfrak{q}}}(X)=X+\delta_{\overline{\mathfrak{n}}}(X)$ for all $X \in \mathfrak{g}^{\prime}$. It is easy to see that $\eta_{\overline{\mathfrak{q}}}=\eta_{\mathfrak{q}}{ }^{\vee}$ and, hence,

$$
\xi^{\vee}=\left(\eta_{\mathfrak{q}} \circ \tilde{\xi}\right)^{\vee}=\eta_{\overline{\mathfrak{q}}} \circ \tilde{\xi}^{\vee} .
$$

Define an algebra homomorphism

$$
\begin{aligned}
\eta_{0}: \mathrm{U}\left(\mathfrak{g}^{\prime} \times \mathfrak{g}^{\prime}\right)=\mathrm{U}\left(\mathfrak{g}^{\prime}\right) \otimes \mathrm{U}\left(\mathfrak{g}^{\prime}\right) & \rightarrow \mathrm{U}\left(\mathfrak{g}^{\prime} \times \mathfrak{g}^{\prime}\right)=\mathrm{U}\left(\mathfrak{g}^{\prime}\right) \otimes \mathrm{U}\left(\mathfrak{g}^{\prime}\right), \\
X^{\prime} \otimes Y^{\prime} & \mapsto \eta_{\overline{\mathfrak{q}}}\left(X^{\prime}\right) \otimes \eta_{\mathfrak{q}}\left(Y^{\prime}\right) .
\end{aligned}
$$

We only need to show that

$$
T_{Z}^{\prime}\left(\delta_{\mathfrak{n}}^{+} \phi\right)=\delta_{\mathfrak{n}}^{+} T_{\eta_{0}(Z)}^{\prime}(\phi)
$$

for all $Z \in \mathrm{U}\left(\mathfrak{g}^{\prime} \times \mathfrak{g}^{\prime}\right)$. As $\mathrm{U}\left(\mathfrak{g}^{\prime} \times \mathfrak{g}^{\prime}\right)$ is generated by $\mathfrak{g}^{\prime} \times \mathfrak{g}^{\prime}$, it is sufficient to show that the above equality holds for $Z \in \mathfrak{g}^{\prime} \times \mathfrak{g}^{\prime}$. Now assume $Z=\left(X^{\prime}, Y^{\prime}\right) \in \mathfrak{g}^{\prime} \times \mathfrak{g}^{\prime}$. Then $T_{Z}^{\prime}$ is an action defined by a vector field on $G_{\mathbb{C}}^{\prime} / U^{\prime}$. Hence,

$$
T_{Z}^{\prime}\left(\delta_{\mathfrak{n}}^{+} \phi\right)=T_{Z}^{\prime}\left(\delta_{\mathfrak{n}}^{+}\right) \phi+\delta_{\mathfrak{n}}^{+} T_{Z}^{\prime}(\phi)
$$

We easily find that

$$
T_{Z}^{\prime}\left(\delta_{\mathfrak{n}}^{+}\right)=\left(\delta_{\overline{\mathfrak{n}}}\left(X^{\prime}\right)+\delta_{\mathfrak{n}}\left(Y^{\prime}\right)\right) \delta_{\mathfrak{n}}^{+}
$$

Therefore,

$$
\begin{aligned}
T_{Z}^{\prime}\left(\delta_{\mathfrak{n}}^{+} \phi\right) & =T_{Z}^{\prime}\left(\delta_{\mathfrak{n}}^{+}\right) \phi+\delta_{\mathfrak{n}}^{+} T_{Z}^{\prime}(\phi) \\
& =\delta_{\mathfrak{n}}^{+}\left(\left(\delta_{\overline{\mathfrak{n}}}\left(X^{\prime}\right)+\delta_{\mathfrak{n}}\left(Y^{\prime}\right)\right) \phi+T_{Z}^{\prime} \phi\right) \\
& =\delta_{\mathfrak{n}}^{+} T_{\eta_{0}(Z)}^{\prime}(\phi) .
\end{aligned}
$$

Proof of Theorem 4.1. Denote by $T_{k}: G_{\mathbb{C}} / U \rightarrow G_{\mathbb{C}} / U$ the translation by $k$ for all $k \in K$. Let $\epsilon_{\mathbf{V}}$ be the embedding of $\mathbf{V}^{\prime}$ into $\mathbf{V}$. Write

$$
f=\epsilon_{\mathbf{V}} \circ\left(\delta_{\mathfrak{n}}^{+} \phi\right) \circ H_{\mathfrak{q}} \in \mathrm{C}^{\infty}\left(G_{\mathbb{C}} / U ; \mathbf{V}\right) .
$$

Then

Note that

$$
E_{\mathfrak{q}}(\phi)=\left.\int_{K} \tau\right|_{K}\left(k^{-1}\right) \circ f \circ T_{k} d k
$$

$$
T_{X \otimes Y}: \mathrm{C}^{\infty}\left(G_{\mathbb{C}} / U ; \mathbf{V}\right) \rightarrow \mathrm{C}^{\infty}\left(G_{\mathbb{C}} / U ; \mathbf{V}\right)
$$




\section{MATRIX COEFFICIENTS}

is a differential operator. Therefore, we have

$$
\begin{aligned}
T_{X \otimes Y} E_{\mathfrak{q}}(\phi) & =\int_{K} T_{X \otimes Y}\left(\left.\tau\right|_{K}\left(k^{-1}\right) \circ f \circ T_{k}\right) d k \\
& =\left.\int_{K} \tau\right|_{K}\left(k^{-1}\right) \circ T_{X \otimes Y}\left(f \circ T_{k}\right) d k .
\end{aligned}
$$

Since $X \otimes Y \in \mathrm{U}(\mathfrak{g})^{K} \otimes \mathrm{U}(\mathfrak{g})^{K}$, we have

$$
T_{X \otimes Y}\left(f \circ T_{k}\right)=T_{X \otimes Y}(f) \circ T_{k} .
$$

Hence,

$$
T_{X \otimes Y} E_{\mathfrak{q}}(\phi)=\left.\int_{K} \tau\right|_{K}\left(k^{-1}\right) \circ T_{X \otimes Y}(f) \circ T_{k} d k .
$$

Note that the map

$$
\begin{aligned}
\mathrm{C}^{\infty}\left(G_{\mathbb{C}}^{\prime} / U^{\prime} ; \mathbf{V}^{\prime}\right) & \rightarrow \mathrm{C}^{\infty}\left(G_{\mathbb{C}} / U ; \mathbf{V}\right) \\
\psi & \mapsto \epsilon_{\mathbf{V}} \circ \psi \circ H_{\mathfrak{q}}
\end{aligned}
$$

is $G_{\mathbb{C}^{-}}^{\prime}$-equivariant. Therefore,

$$
T_{X^{\prime} \otimes Y^{\prime}}\left(\epsilon_{\mathbf{V}} \circ \psi \circ H_{\mathfrak{q}}\right)=\epsilon_{\mathbf{V}} \circ T_{X^{\prime} \otimes Y^{\prime}}^{\prime} \psi \circ H_{\mathfrak{q}}
$$

for all $X^{\prime} \otimes Y^{\prime} \in \mathrm{U}\left(\mathfrak{g}^{\prime}\right) \otimes \mathrm{U}\left(\mathfrak{g}^{\prime}\right), \psi \in \mathrm{C}^{\infty}\left(G_{\mathbb{C}}^{\prime} / U^{\prime} ; \mathbf{V}^{\prime}\right)$.

Now we have

$$
\begin{aligned}
T_{X \otimes Y}(f) & =T_{\tilde{\xi} \vee(X) \otimes \tilde{\xi}(Y)} f \quad \text { by Lemma } 4.2 \\
& =T_{\tilde{\xi} \vee}(X) \otimes \tilde{\xi}(Y) \\
& =\epsilon_{\mathbf{V}} \circ\left(\epsilon_{\tilde{\xi} \vee}^{\prime} \circ\left(\delta_{\mathfrak{n}}^{+} \phi\right) \circ H_{\mathfrak{q}}\right) \\
& =\epsilon_{\mathbf{V}} \circ\left(\delta_{\mathfrak{n}}^{+} T_{\xi^{\vee}(X) \otimes \xi(Y)}^{\prime}\left(\delta_{\mathfrak{n}}^{+} \phi\right)\right) \circ H_{\mathfrak{q}} \quad \text { by }(17)
\end{aligned}
$$

In conclusion,

$$
\begin{aligned}
& T_{X \otimes Y} E_{\mathfrak{q}}(\phi)=\left.\int_{K} \tau\right|_{K}\left(k^{-1}\right) \circ T_{X \otimes Y}(f) \circ T_{k} d k
\end{aligned}
$$

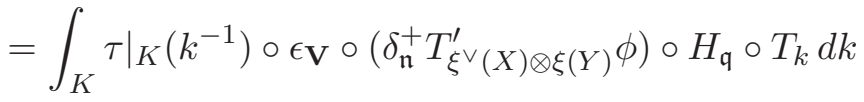

$$
\begin{aligned}
& =E_{\mathfrak{q}}\left(T_{\xi^{\vee}}^{\prime}(X) \otimes \xi(Y) \phi\right) \text {. }
\end{aligned}
$$

\section{Cohomological inductions and bottom layers}

We call a vector space a weak $\left(\mathrm{U}(\mathfrak{g})^{K}, K\right)$-module if it is a locally finite $K$-module as well as a $\mathrm{U}(\mathfrak{g})^{K}$ module such that the $K$-action and the $\mathrm{U}(\mathfrak{g})^{K}$-action commute. Note that the $\alpha$-isotypic component $M(\alpha)$ is a weak $\left(\mathrm{U}(\mathfrak{g})^{K}, K\right)$-module for every $(\mathfrak{g}, K)$-module $M$ and every $K$-type $\alpha$. In this section, we determine the weak $\left(\mathrm{U}(\mathfrak{g})^{K}, K\right)$-module structure of a bottom layer of a cohomologically induced representation.

\subsection{Zuckerman functors}

Let us recall cohomological inductions and bottom layer maps from [KV95]. We continue to use the notation of $\S 3.1$. 


\section{B. SUN}

Let $\Gamma^{0}$ be the Zuckerman functor from the category of $\left(\mathfrak{g}, K^{\prime}\right)$-modules to the category of $(\mathfrak{g}, K)$-modules. It is given by

$$
\begin{gathered}
\Gamma^{0}(M)=\text { sum of all finite-dimensional } \mathfrak{k} \text { invariant subspaces of } M \\
\text { for which the actions of } \mathfrak{k} \text { globalize to } K .
\end{gathered}
$$

This is a left exact covariant functor. Denote by $\Gamma^{i}$ its $i$ th right derived functor, $i=0,1,2, \ldots$. Let $\Gamma_{K}^{0}$ be the Zuckerman functor from the category of $\left(\mathfrak{k}, K^{\prime}\right)$-modules to the category of $(\mathfrak{k}, K)$-modules. Denote by $\Gamma_{K}^{i}$ its $i$ th right derived functor, $i=0,1,2, \ldots$.

Let $\operatorname{pro}_{\mathfrak{q}, K^{\prime}}^{\mathfrak{g}, K^{\prime}}$ be the exact covariant functor from the category of $\left(\mathfrak{q}, K^{\prime}\right)$-modules to the category of $\left(\mathfrak{g}, K^{\prime}\right)$-modules given by

$$
\operatorname{pro}_{\mathfrak{q}, K^{\prime}}^{\mathfrak{g}, K^{\prime}}(M)=\operatorname{Hom}_{\mathrm{U}(\mathfrak{q})}(\mathrm{U}(\mathfrak{g}), M)_{K^{\prime}},
$$

where the $\mathrm{U}(\mathfrak{g})$-action on $\operatorname{Hom}_{\mathrm{U}(\mathfrak{q})}(\mathrm{U}(\mathfrak{g}), M)$ is given by

$$
(X \phi)(u)=\phi(u X), \quad X, u \in \mathrm{U}(\mathfrak{g}), \phi \in \operatorname{Hom}_{\mathrm{U}(\mathfrak{q})}(\mathrm{U}(\mathfrak{g}), M),
$$

the $K^{\prime}$-action on $\operatorname{Hom}_{\mathrm{U}(\mathfrak{q})}(\mathrm{U}(\mathfrak{g}), M)$ is given by

$$
(k \phi)(u)=k\left(\phi\left(\operatorname{Ad}_{k^{-1}} u\right)\right), \quad k \in K^{\prime}, u \in \mathrm{U}(\mathfrak{g}), \phi \in \operatorname{Hom}_{\mathrm{U}(\mathfrak{q})}(\mathrm{U}(\mathfrak{g}), M),
$$

and

$$
\operatorname{Hom}_{\mathrm{U}(\mathfrak{q})}(\mathrm{U}(\mathfrak{g}), M)_{K^{\prime}} \text { is the } K^{\prime} \text {-finite vectors of } \operatorname{Hom}_{\mathrm{U}\left(\mathfrak{g}^{\prime}\right)}(\mathrm{U}(\mathfrak{g}), M) \text {. }
$$

Let $\operatorname{pro}_{\mathfrak{q} \cap \mathfrak{k}, K^{\prime}}^{\mathfrak{k}, K^{\prime}}$ be the analogous exact covariant functor from the category of $\left(\mathfrak{q} \cap \mathfrak{k}, K^{\prime}\right)$-modules to the category of $\left(\mathfrak{k}, K^{\prime}\right)$-modules. Let $\mathcal{F}$ denote the forgetful functors in various contexts. For example, $\mathcal{F}_{\mathfrak{g}^{\prime}, K^{\prime}}^{\mathfrak{q}, K^{\prime}}$ is the forgetful functor from the category of $\left(\mathfrak{g}^{\prime}, K^{\prime}\right)$-modules to the category of $\left(\mathfrak{q}, K^{\prime}\right)$-modules via trivial $\mathfrak{n}$-action.

Let $M^{\prime}$ be a $\left(\mathfrak{g}^{\prime}, K^{\prime}\right)$-module and define another $\left(\mathfrak{g}^{\prime}, K^{\prime}\right)$-module

$$
M^{\prime \#}=M^{\prime} \otimes \wedge^{\text {top }} \mathfrak{n} .
$$

Define

$$
\mathcal{R}^{i}\left(M^{\prime}\right)=\left(\Gamma^{i} \circ \operatorname{pro}_{\mathfrak{q}, K^{\prime}}^{\mathfrak{g}, K^{\prime}} \circ \mathcal{F}_{\mathfrak{g}^{\prime}, K^{\prime}}^{\mathfrak{q}, K^{\prime}}\right)\left(M^{\prime \#}\right) .
$$

Then $\mathcal{R}^{i}$ is the cohomological induction functor from the category of $\left(\mathfrak{g}^{\prime}, K^{\prime}\right)$-modules to the category of $(\mathfrak{g}, K)$-modules.

Define

$$
\mathcal{R}_{\circ}^{i}\left(M^{\prime}\right)=\left(\Gamma_{K}^{i} \circ \mathcal{F}_{\mathfrak{g}, K^{\prime}}^{\mathfrak{k}, K^{\prime}} \circ \operatorname{pro}_{\mathfrak{q}, K^{\prime}}^{\mathfrak{g}, K^{\prime}} \circ \mathcal{F}_{\mathfrak{g}^{\prime}, K^{\prime}}^{\mathfrak{q}, K^{\prime}}\right)\left(M^{\prime \#}\right)
$$

Note that

$$
\mathrm{U}(\mathfrak{g})^{K} \quad \operatorname{acts} \text { on }\left(\mathcal{F}_{\mathfrak{g}, K^{\prime}}^{\mathfrak{k}, K^{\prime}} \circ \operatorname{pro}_{\mathfrak{q}, K^{\prime}}^{\mathfrak{g}, K^{\prime}} \circ \mathcal{F}_{\mathfrak{g}^{\prime}, K^{\prime}}^{\mathfrak{q}, K^{\prime}}\right)\left(M^{\prime \#}\right)
$$

by $\left(\mathfrak{k}, K^{\prime}\right)$-module endomorphisms. By using the functor $\Gamma_{K}^{i}$, we get a $\mathrm{U}(\mathfrak{g})^{K}$-action on $\mathcal{R}_{\circ}^{i}\left(M^{\prime}\right)$ by $(\mathfrak{k}, K)$-module endomorphisms.

Note that

$$
\mathfrak{k}^{\prime}=\operatorname{Lie}\left(K^{\prime}\right) \otimes_{\mathbb{R}} \mathbb{C}=\mathfrak{g}^{\prime} \cap \mathfrak{k} \quad \text { and } \quad \mathfrak{q} \cap \mathfrak{k}=\mathfrak{k}^{\prime} \oplus(\mathfrak{n} \cap \mathfrak{k}) .
$$

Define

where

$$
\mathcal{R}_{K}^{i}\left(M^{\prime}\right)=\left(\Gamma_{K}^{i} \circ \operatorname{pro}_{\mathfrak{q} \cap \mathfrak{k}, K^{\prime}}^{\mathfrak{k}, K^{\prime}} \circ \mathcal{F}_{\mathfrak{g}^{\prime}, K^{\prime}}^{\mathfrak{q} \cap \mathfrak{k}, K^{\prime}}\right)\left(M^{\prime \#}\right),
$$

$$
\mathcal{F}_{\mathfrak{g}^{\prime}, K^{\prime}}^{\mathfrak{q} \cap K^{\prime}}=\mathcal{F}_{\mathfrak{k}^{\prime}, K^{\prime}}^{\mathfrak{q} \cap \mathfrak{k}, K^{\prime}} \circ \mathcal{F}_{\mathfrak{g}^{\prime}, K^{\prime}}^{\mathfrak{k}^{\prime}, K^{\prime}}
$$




\section{Matrix COEFFicients}

and $\mathcal{F}_{\mathfrak{k}^{\prime}, K^{\prime}}^{\mathfrak{q} \cap \mathfrak{k}, K^{\prime}}$ is defined via the trivial $\mathfrak{n} \cap \mathfrak{k}$-action. We have a $\mathrm{U}\left(\mathfrak{g}^{\prime}\right)^{K^{\prime}}$-action on $\mathcal{F}_{\mathfrak{g}^{\prime}, K^{\prime}}^{\mathfrak{q} \cap \mathfrak{k}, K^{\prime}}\left(M^{\prime \#}\right)$ by $\left(\mathfrak{q} \cap \mathfrak{k}, K^{\prime}\right)$-module endomorphisms. By applying the functor $\operatorname{pro}_{\mathfrak{q} \cap \mathfrak{k}, K^{\prime}}^{\mathfrak{k}, K^{\prime}}$, we get a $\mathrm{U}\left(\mathfrak{g}^{\prime}\right)^{K^{\prime}}$-action on ( $\left.\operatorname{pro}_{\mathfrak{q} \cap \mathfrak{k}, K^{\prime}}^{\mathfrak{k}, K^{\prime}} \circ \mathcal{F}_{\mathfrak{g}^{\prime}, K^{\prime}}^{\mathfrak{q} \mathfrak{k}, K^{\prime}}\right)\left(M^{\prime \#}\right)$ by $\left(\mathfrak{k}, K^{\prime}\right)$-module endomorphisms. Then, by applying $\Gamma_{K}^{i}$, we get a $\mathrm{U}\left(\mathfrak{g}^{\prime}\right)^{K^{\prime}}$-action on $\mathcal{R}_{K}^{i}\left(M^{\prime}\right)$ by $(\mathfrak{k}, K)$-module endomorphisms.

Lemma 5.1. For any $\left(\mathfrak{g}^{\prime}, K^{\prime}\right)$-module $M^{\prime}, \mathcal{R}^{i}\left(M^{\prime}\right)$ is canonically isomorphic to $\mathcal{R}_{\circ}^{i}\left(M^{\prime}\right)$ as a weak $\left(\mathrm{U}(\mathfrak{g})^{K}, K\right)$-module.

Proof. Write $M^{\prime \prime}=\left(\operatorname{pro}_{\mathfrak{q}, K^{\prime}}^{\mathfrak{g}, K^{\prime}} \circ \mathcal{F}_{\mathfrak{g}^{\prime}, K^{\prime}}^{\mathfrak{q}, K^{\prime}}\right)\left(M^{\prime \#}\right)$ and let

$$
0 \rightarrow M^{\prime \prime} \rightarrow J_{0} \rightarrow J_{1} \rightarrow J_{2} \rightarrow \cdots
$$

be an injective resolution of $M^{\prime \prime}$ in the category of $\left(\mathfrak{g}, K^{\prime}\right)$-modules. By [KV95, Proposition 2.57], the exact functor $\mathcal{F}_{\mathfrak{g}, K^{\prime}}^{\mathfrak{k}, K^{\prime}}$ sends injectives to injectives. Therefore,

$$
0 \rightarrow \mathcal{F}_{\mathfrak{g}, K^{\prime}}^{\mathfrak{k}, K^{\prime}}\left(M^{\prime \prime}\right) \rightarrow \mathcal{F}_{\mathfrak{g}, K^{\prime}}^{\mathfrak{k}, K^{\prime}}\left(J_{0}\right) \rightarrow \mathcal{F}_{\mathfrak{g}, K^{\prime}}^{\mathfrak{k}, K^{\prime}}\left(J_{1}\right) \rightarrow \mathcal{F}_{\mathfrak{g}, K^{\prime}}^{\mathfrak{k}, K^{\prime}}\left(J_{2}\right) \rightarrow \cdots
$$

is an injective resolution of $\mathcal{F}_{\mathfrak{g}, K^{\prime}}^{\mathfrak{k}, K^{\prime}}\left(M^{\prime \prime}\right)$ in the category of $\left(\mathfrak{k}, K^{\prime}\right)$-modules.

Note that

$$
0 \rightarrow \Gamma^{0}\left(J_{0}\right) \rightarrow \Gamma^{0}\left(J_{1}\right) \rightarrow \Gamma^{0}\left(J_{2}\right) \rightarrow \cdots
$$

and

$$
0 \rightarrow \Gamma_{K}^{0}\left(\mathcal{F}_{\mathfrak{g}, K^{\prime}}^{\mathfrak{k}, K^{\prime}}\left(J_{0}\right)\right) \rightarrow \Gamma_{K}^{0}\left(\mathcal{F}_{\mathfrak{g}, K^{\prime}}^{\mathfrak{k}, K^{\prime}}\left(J_{1}\right)\right) \rightarrow \Gamma_{K}^{0}\left(\mathcal{F}_{\mathfrak{g}, K^{\prime}}^{\mathfrak{k}, K^{\prime}}\left(J_{2}\right)\right) \rightarrow \cdots
$$

are exactly the same as sequences of weak $\left(\mathrm{U}(\mathfrak{g})^{K}, K\right)$-modules. Taking the $i$ th cohomology of both sequences, we get $\mathcal{R}^{i}\left(M^{\prime}\right)=\mathcal{R}_{\circ}^{i}\left(M^{\prime}\right)$ as a weak $\left(\mathrm{U}(\mathfrak{g})^{K}, K\right)$-module.

\subsection{Bottom layer maps}

Let $M^{\prime}$ be a $\left(\mathfrak{g}^{\prime}, K^{\prime}\right)$-module. Define a $\left(\mathfrak{k}, K^{\prime}\right)$-module homomorphism

$$
\beta_{M^{\prime}}:\left(\mathcal{F}_{\mathfrak{g}, K^{\prime}}^{\mathfrak{k}, K^{\prime}} \circ \operatorname{pro}_{\mathfrak{q}, K^{\prime}}^{\mathfrak{g}, K^{\prime}} \circ \mathcal{F}_{\mathfrak{g}^{\prime}, K^{\prime}}^{\mathfrak{q}, K^{\prime}}\right)\left(M^{\prime \#}\right) \rightarrow\left(\operatorname{pro}_{\mathfrak{q} \cap \mathfrak{k}, K^{\prime}}^{\mathfrak{k}, K^{\prime}} \circ \mathcal{F}_{\mathfrak{g}^{\prime}, K^{\prime}}^{\mathfrak{q} \cap \mathfrak{k}, K^{\prime}}\right)\left(M^{\prime \#}\right)
$$

by

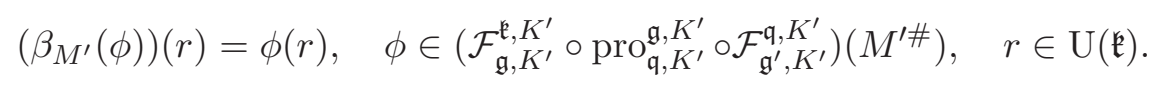

Lemma 5.2. For all $X \in \mathrm{U}(\mathfrak{g})^{K}$, the following diagram commutes.

$$
\begin{aligned}
& \left(\mathcal{F}_{\mathfrak{g}, K^{\prime}}^{\mathfrak{k}, K^{\prime}} \circ \operatorname{pro}_{\mathfrak{q}, K^{\prime}}^{\mathfrak{g}, K^{\prime}} \circ \mathcal{F}_{\mathfrak{g}^{\prime}, K^{\prime}}^{\mathfrak{q}, K^{\prime}}\right)\left(M^{\prime \#}\right) \stackrel{\beta_{M^{\prime}}}{\longrightarrow}\left(\operatorname{pro}_{\mathfrak{q} \cap \mathfrak{k}, K^{\prime}}^{\mathfrak{k}, K^{\prime}} \circ \mathcal{F}_{\mathfrak{g}^{\prime}, K^{\prime}}^{\mathfrak{q} \cap \mathfrak{k}, K^{\prime}}\right)\left(M^{\prime \#}\right) \\
& X \downarrow \tilde{\xi}(X) \downarrow \\
& \left(\mathcal{F}_{\mathfrak{g}, K^{\prime}}^{\mathfrak{k}, K^{\prime}} \circ \operatorname{pro}_{\mathfrak{q}, K^{\prime}}^{\mathfrak{g}, K^{\prime}} \circ \mathcal{F}_{\mathfrak{g}^{\prime}, K^{\prime}}^{\mathfrak{q}, K^{\prime}}\right)\left(M^{\prime \#}\right) \stackrel{\beta_{M^{\prime}}}{\longrightarrow}\left(\operatorname{pro}_{\mathfrak{q} \cap \mathfrak{k}, K^{\prime}}^{\mathfrak{k}, K^{\prime}} \circ \mathcal{F}_{\mathfrak{g}^{\prime}, K^{\prime}}^{\mathfrak{q} \cap \mathfrak{k}, K^{\prime}}\right)\left(M^{\prime \#}\right)
\end{aligned}
$$

Proof. Let

$$
X=a X_{1}+X_{2}
$$

where $a \in \mathfrak{n}, X_{1} \in \mathrm{U}(\mathfrak{g})$, and

$$
X_{2}=\tilde{\xi}(X) \in \mathrm{U}\left(\mathfrak{g}^{\prime}\right)^{K^{\prime}}
$$

Let

$$
\phi \in\left(\mathcal{F}_{\mathfrak{g}, K^{\prime}}^{\mathfrak{k}, K^{\prime}} \circ \operatorname{pro}_{\mathfrak{q}, K^{\prime}}^{\mathfrak{g}, K^{\prime}} \circ \mathcal{F}_{\mathfrak{g}^{\prime}, K^{\prime}}^{\mathfrak{q}, K^{\prime}}\right)\left(M^{\prime \#}\right) \subset \operatorname{Hom}_{\mathrm{U}(\mathfrak{q})}\left(\mathrm{U}(\mathfrak{g}), M^{\prime \#}\right)
$$


and $r \in \mathrm{U}(\mathfrak{k})$. We have

$$
\begin{aligned}
\left(\beta_{M^{\prime}}(X \phi)\right)(r) & =(X \phi)(r)=\phi(r X)=\phi(X r) \\
& =\phi\left(a X_{1} r\right)+\phi\left(X_{2} r\right) \\
& =a\left(\phi\left(X_{1}\right)\right)+X_{2}(\phi(r)) \\
& =X_{2}\left(\beta_{M^{\prime}}(\phi)(r)\right) \\
& =\tilde{\xi}(X)\left(\beta_{M^{\prime}}(\phi)\right)(r) .
\end{aligned}
$$

Therefore,

$$
\beta_{M^{\prime}}(X \phi)=\tilde{\xi}(X)\left(\beta_{M^{\prime}}(\phi)\right)
$$

By applying the functor $\Gamma_{K}^{i}$ to $\beta_{M^{\prime}}$, we define the bottom layer map

$$
\mathcal{B}_{M^{\prime}}=\Gamma_{K}^{i}\left(\beta_{M^{\prime}}\right): \mathcal{R}_{\circ}^{i}\left(M^{\prime}\right) \rightarrow \mathcal{R}_{K}^{i}\left(M^{\prime}\right)
$$

which is a $(\mathfrak{k}, K)$-module homomorphism. Applying $\Gamma_{K}^{i}$ to the commutative diagram of the above lemma, we obtain the following.

Proposition 5.3. For all $X \in \mathrm{U}(\mathfrak{g})^{K}$, the following diagram commutes.

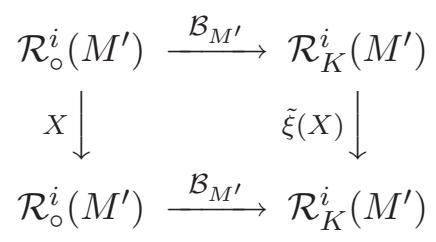

\subsection{Bottom layers}

From now on we fix a $K$-type $\alpha$. We also fix an irreducible representation $\sigma_{0}$ of $K$ of type $\alpha$ on a vector space $W_{\alpha}$. Let $\sigma_{0}^{\prime}$ be the representation of $K^{\prime}$ on $W_{\alpha^{\prime}}$ which is q-associated to $\sigma_{0}$ (Definition 3.1). The representation $\sigma_{0}^{\prime}$ is also irreducible by the highest weight theory. We call the $K^{\prime}$-type $\alpha^{\prime}$ of $\sigma_{0}^{\prime}$ the $K^{\prime}$-type $\mathfrak{q}$-associated to $\alpha$.

Set $S=\operatorname{dim}(\mathfrak{n} \cap \mathfrak{k})$ as usual. The most interesting case of cohomological induction is that when $i=S$. Write

$$
M=\mathcal{R}^{S}\left(M^{\prime}\right) \text { and } L=\operatorname{Hom}_{K^{\prime}}\left(W_{\alpha^{\prime}}, M^{\prime}\right) .
$$

Then $L$ is a $\mathrm{U}\left(\mathfrak{g}^{\prime}\right)^{K^{\prime}}$-module by the action on $M^{\prime}$. We make $L$ a $\mathrm{U}(\mathfrak{g})^{K}$-module by the formula

$$
X v=\xi(X) v, \quad X \in \mathrm{U}(\mathfrak{g})^{K}, v \in L .
$$

Now $L \otimes W_{\alpha}$ is a weak $\left(\mathrm{U}(\mathfrak{g})^{K}, K\right)$-module by the action of $\mathrm{U}(\mathfrak{g})^{K}$ on the first factor, and the action of $K$ on the second factor.

Definition 5.4. Let $M^{\prime}$ be a $\left(\mathfrak{g}^{\prime}, K^{\prime}\right)$-module, $M=\mathcal{R}^{S}\left(M^{\prime}\right)$. The $K$-type $\alpha$ is said to be in the bottom layer of $M$ if the map induced by $\mathcal{B}_{M^{\prime}}$,

$$
\mathcal{B}_{M^{\prime}}(\alpha): M(\alpha)=\mathcal{R}_{\circ}^{S}\left(M^{\prime}\right)(\alpha) \rightarrow \mathcal{R}_{K}^{S}\left(M^{\prime}\right)(\alpha)
$$

is bijective and nonzero.

We know that under a weak condition on $M^{\prime}, \alpha$ is in the bottom layer of $M$ if and only if $M^{\prime}\left(\alpha^{\prime}\right) \neq 0$. The condition holds when $M^{\prime}$ has an infinitesimal character [KV95, Theorem 5.80, Corollary 5.72]. The main result of this section is the following theorem.

TheOREm 5.5. With the notation as above, if $\alpha$ is in the bottom layer of $M$, then $M(\alpha)$ is isomorphic to $L \otimes W_{\alpha}$ as a weak $\left(\mathrm{U}(\mathfrak{g})^{K}, K\right)$-module. 


\section{MATRIX COEFFICIENTS}

Proof. We easily check that the diagram

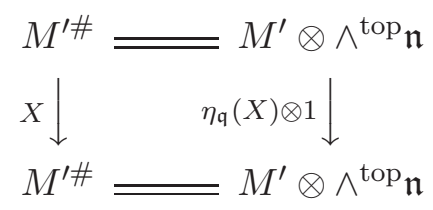

commutes for every $X \in \mathrm{U}\left(\mathfrak{g}^{\prime}\right)$, where $\eta_{\mathfrak{q}}$ is defined in (16). This implies the diagram

$$
\begin{aligned}
\operatorname{Hom}_{K^{\prime}}\left(W_{\alpha^{\prime}} \otimes \wedge^{\text {top }} \mathfrak{n}, M^{\prime \#}\right) & =\operatorname{Hom}_{K^{\prime}}\left(W_{\alpha^{\prime}} \otimes \wedge^{\text {top }} \mathfrak{n}, M^{\prime} \otimes \wedge^{\text {top }} \mathfrak{n}\right) \\
X & \eta_{\mathfrak{q}}(X) \otimes 1 \\
\operatorname{Hom}_{K^{\prime}}\left(W_{\alpha^{\prime}} \otimes \wedge^{\text {top }} \mathfrak{n}, M^{\prime \#}\right) & =\operatorname{Hom}_{K^{\prime}}\left(W_{\alpha^{\prime}} \otimes \wedge^{\text {top }} \mathfrak{n}, M^{\prime} \otimes \wedge^{\text {top }} \mathfrak{n}\right)
\end{aligned}
$$

commutes for every $X \in \mathrm{U}\left(\mathfrak{g}^{\prime}\right)^{K^{\prime}}$. It is obvious that the diagram

$$
\begin{array}{rr}
\operatorname{Hom}_{K^{\prime}}\left(W_{\alpha^{\prime}} \otimes \wedge^{\text {top }} \mathfrak{n}, M^{\prime} \otimes \wedge^{\text {top }} \mathfrak{n}\right) & =\operatorname{Hom}_{K^{\prime}}\left(W_{\alpha^{\prime}}, M^{\prime}\right) \\
\eta_{\mathfrak{q}}(X) \otimes 1 & \eta_{\mathfrak{q}}(X) \downarrow \\
\operatorname{Hom}_{K^{\prime}}\left(W_{\alpha^{\prime}} \otimes \wedge^{\text {top }} \mathfrak{n}, M^{\prime} \otimes \wedge^{\text {top }} \mathfrak{n}\right) & =\operatorname{Hom}_{K^{\prime}}\left(W_{\alpha^{\prime}}, M^{\prime}\right)
\end{array}
$$

commutes for every $X \in \mathrm{U}\left(\mathfrak{g}^{\prime}\right)^{K^{\prime}}$. By combining these two diagrams, and replacing $X$ by $\tilde{\xi}(X)$, we get a commutative diagram

$$
\begin{array}{rc}
\operatorname{Hom}_{K^{\prime}}\left(W_{\alpha^{\prime}} \otimes \wedge^{\text {top }} \mathfrak{n}, M^{\prime \#}\right) & =\operatorname{Hom}_{K^{\prime}}\left(W_{\alpha^{\prime}}, M^{\prime}\right) \\
\tilde{\xi}(X) \downarrow & \xi(X) \downarrow \\
\operatorname{Hom}_{K^{\prime}}\left(W_{\alpha^{\prime}} \otimes \wedge^{\text {top }} \mathfrak{n}, M^{\prime \#}\right) & =\operatorname{Hom}_{K^{\prime}}\left(W_{\alpha^{\prime}}, M^{\prime}\right)
\end{array}
$$

for every $X \in \mathrm{U}(\mathfrak{g})^{K}$.

Now (11) implies

$$
\mathrm{H}^{0}\left(\mathfrak{n} \cap \mathfrak{k}, W_{\alpha}\right) \otimes \wedge^{\text {top }}(\mathfrak{n} \cap \mathfrak{k}) \cong W_{\alpha^{\prime}} \otimes \wedge^{\text {top }_{\mathfrak{n}}}
$$

as a $K^{\prime}$-module. Theorem 4.155 of [KV95] states that there is a natural isomorphism

$$
\operatorname{Hom}_{K}\left(W_{\alpha}, \mathcal{R}_{K}^{S}\left(M^{\prime}\right)\right) \cong \operatorname{Hom}_{K^{\prime}}\left(\mathrm{H}^{0}\left(\mathfrak{n} \cap \mathfrak{k}, W_{\alpha}\right) \otimes \wedge^{\text {top }}(\mathfrak{n} \cap \mathfrak{k}), M^{\prime \#}\right)
$$

The isomorphism (19) and the naturalness of (20) imply that we actually have a $\mathrm{U}\left(\mathfrak{g}^{\prime}\right)^{K^{\prime}}$-module isomorphism

$$
\operatorname{Hom}_{K}\left(W_{\alpha}, \mathcal{R}_{K}^{S}\left(M^{\prime}\right)\right) \cong \operatorname{Hom}_{K^{\prime}}\left(W_{\alpha^{\prime}} \otimes \wedge^{\text {top }} \mathfrak{n}, M^{\prime \#}\right) .
$$

Lemma 5.1 and Proposition 5.3 give a commutative diagram

$$
\begin{aligned}
\operatorname{Hom}_{K}\left(W_{\alpha}, \mathcal{R}^{S}\left(M^{\prime}\right)\right) & \longrightarrow \operatorname{Hom}_{K}\left(W_{\alpha}, \mathcal{R}_{K}^{S}\left(M^{\prime}\right)\right) \\
X \downarrow & \tilde{\xi}(X) \downarrow \\
\operatorname{Hom}_{K}\left(W_{\alpha}, \mathcal{R}^{S}\left(M^{\prime}\right)\right) & \longrightarrow \operatorname{Hom}_{K}\left(W_{\alpha}, \mathcal{R}_{K}^{S}\left(M^{\prime}\right)\right)
\end{aligned}
$$

for every $X \in \mathrm{U}(\mathfrak{g})^{K}$, where each row is the map induced by $\mathcal{B}_{M^{\prime}}$, which is an isomorphism as $\alpha$ is in the bottom layer. We put the last two commutative diagrams and the isomorphism (21) together, and obtain a commutative diagram

$$
\begin{aligned}
& \operatorname{Hom}_{K}\left(W_{\alpha}, \mathcal{R}^{S}\left(M^{\prime}\right)\right) \longrightarrow L=\operatorname{Hom}_{K^{\prime}}\left(W_{\alpha^{\prime}}, M^{\prime}\right) \\
& X \downarrow \xi(X) \downarrow \\
& \operatorname{Hom}_{K}\left(W_{\alpha}, \mathcal{R}^{S}\left(M^{\prime}\right)\right) \longrightarrow L=\operatorname{Hom}_{K^{\prime}}\left(W_{\alpha^{\prime}}, M^{\prime}\right)
\end{aligned}
$$

for every $X \in \mathrm{U}(\mathfrak{g})^{K}$, with bijective horizontal maps. This finishes the proof. 


\section{B. SUN}

\section{Matrix coefficients of cohomologically induced representations}

In this section, we use the results of the previous sections to find integral representations of matrix coefficients of bottom layers of cohomologically induced representations.

\subsection{Matrix coefficient of a $K$-type}

Let $\alpha$ be a $K$-type as before. Assume that $M$ is a general finitely generated admissible $(\mathfrak{g}, K)$ module, with the actions $\pi$ of $\mathrm{U}(\mathfrak{g})$ and $K$ on it. Write $W=M(\alpha)$. Then $W$ is a finite-dimensional weak $\left(\mathrm{U}(\mathfrak{g})^{K}, K\right)$-module. We easily check that the function $\phi_{M, \alpha} \in \mathrm{C}^{\omega}\left(G ; \operatorname{End}_{\mathbb{C}}(W)\right)$ defined in the introduction satisfies

$$
\left\{\begin{array}{l}
\text { (i) } \phi_{M, \alpha}(1)=\text { identity map of } W \\
\text { (ii) } \phi_{M, \alpha}(k x l)=\pi(k) \circ \phi_{M, \alpha}(x) \circ \pi(l), \quad x \in G, k, l \in K \\
\text { (iii) } T_{X \otimes Y} \phi_{M, \alpha}(x)=\pi\left(X^{\vee}\right) \circ \phi_{M, \alpha}(x) \circ \pi(Y), \quad x \in G, X \otimes Y \in \mathrm{U}(\mathfrak{g})^{K} \otimes \mathrm{U}(\mathfrak{g})^{K} .
\end{array}\right.
$$

These conditions are generalizations of those of (1) for elementary spherical functions.

Proposition 6.1. Let $W$ be a weak $\left(\mathrm{U}(\mathfrak{g})^{K}, K\right)$-module which is isomorphic to $M(\alpha)$ for some finitely generated admissible $(\mathfrak{g}, K)$-module $M$. Then there is a unique function in $\mathrm{C}^{\omega}\left(G ; \operatorname{End}_{\mathbb{C}}(W)\right)$ which satisfies the three conditions of (22).

The existence is clear. We omit the standard proof of uniqueness (as indicated in the proof of [Fle80a, Lemma 7.6]). Write $\phi_{W}$ for this unique function. Of course,

$$
\phi_{M(\alpha)}=\phi_{M, \alpha}
$$

and the diagram

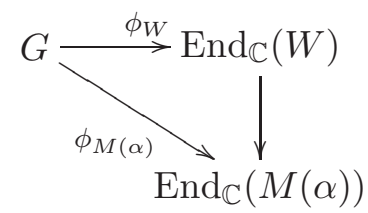

commutes, where the vertical arrow is the linear isomorphism which is induced by an arbitrary $\left(\mathrm{U}(\mathfrak{g})^{K}, K\right)$-isomorphism of $W$ and $M(\alpha)$. Note that if $M$ is irreducible, then $M(\alpha)$ is irreducible as a weak $\left(\mathrm{U}(\mathfrak{g})^{K}, K\right)$-module, and the vertical arrow is uniquely determined.

Write $\psi_{W}, \psi_{M, \alpha} \in \mathrm{C}^{\omega}\left(G_{\mathbb{C}} / U ; \operatorname{End}_{\mathbb{C}}(W)\right)$ for the functions corresponding to $\phi_{W}$ and $\phi_{M, \alpha}$, respectively, in Theorem 2.1. The function $\psi_{W}$ is characterized by

$$
\left\{\begin{array}{l}
\text { (i) } \psi_{W}(1 U)=\text { identity map of } W \\
\text { (ii) } \phi_{W}(k x)=\sigma(k) \circ \phi_{M, \alpha}(x) \circ \sigma\left(\bar{k}^{-1}\right), \quad x \in G_{\mathbb{C}} / U, k \in K_{\mathbb{C}} ; \\
\text { (iii) } T_{X \otimes Y} \phi_{W}(x)=\sigma\left(X^{\vee}\right) \circ \phi_{W}(x) \circ \sigma(Y), \quad x \in G_{\mathbb{C}} / U, X \otimes Y \in \mathrm{U}(\mathfrak{g})^{K} \otimes \mathrm{U}(\mathfrak{g})^{K} ;
\end{array}\right.
$$

where we write $\sigma$ for the actions of $\mathrm{U}(\mathfrak{g})^{K}$ and $K_{\mathbb{C}}$ on $W$. Similarly, we have

$$
\psi_{M(\alpha)}=\psi_{M, \alpha}
$$

and the diagram

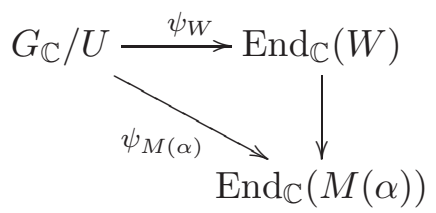

commutes. 


\section{MATRIX COEFFICIENTS}

\subsection{Matrix coefficient of a bottom layer}

Let us return to the situation of $\S 5.3$. Assume that $M^{\prime}$ is admissible, then $M=\mathcal{R}^{S}\left(M^{\prime}\right)$ is also admissible. Assume that $\alpha$ is in the bottom layer of $M$. Write

$$
W^{\prime}=M^{\prime}\left(\alpha^{\prime}\right) \cong L \otimes W_{\alpha^{\prime}}
$$

as weak $\left(\mathrm{U}\left(\mathfrak{g}^{\prime}\right)^{K^{\prime}}, K^{\prime}\right)$-modules. Set

$$
W=L \otimes W_{\alpha}
$$

and view it as a weak $\left(\mathrm{U}(\mathfrak{g})^{K}, K\right)$-module. Theorem 5.5 states that $W \cong M(\alpha)$ as weak $\left(\mathrm{U}(\mathfrak{g})^{K}, K\right)$ modules. Denote by $\sigma$ the actions of $\mathrm{U}(\mathfrak{g})^{K}$ and $K$ on $W$ and by $\sigma^{\prime}$ the actions of $\mathrm{U}\left(\mathfrak{g}^{\prime}\right)^{K^{\prime}}$ and $K^{\prime}$ on $W^{\prime}$.

Define a representation $\tau$ of $K \times K$ on $\operatorname{End}_{\mathbb{C}}(W)$ by

$$
\tau(k, l)(f)=\sigma(k) \circ f \circ \sigma\left(l^{-1}\right), \quad k, l \in K, f \in \operatorname{End}_{\mathbb{C}}(W),
$$

and a representation $\tau^{\prime}$ of $K^{\prime} \times K^{\prime}$ on $\operatorname{End}_{\mathbb{C}}\left(W^{\prime}\right)$ by

$$
\tau^{\prime}(k, l)(f)=\sigma^{\prime}(k) \circ f \circ \sigma^{\prime}\left(l^{-1}\right), \quad k, l \in K^{\prime}, f \in \operatorname{End}_{\mathbb{C}}\left(W^{\prime}\right) .
$$

We have a decomposition

$$
W=W^{\prime} \oplus \sigma(\overline{\mathfrak{n}} \cap \mathfrak{k}) W .
$$

Therefore, we may view an element of $\operatorname{End}_{\mathbb{C}}\left(W^{\prime}\right)$ as an element of $\operatorname{End}_{\mathbb{C}}(W)$ which vanishes on $\sigma(\overline{\mathfrak{n}} \cap \mathfrak{k}) W$. Denote by

$$
j_{\mathfrak{q}}: \operatorname{End}_{\mathbb{C}}\left(W^{\prime}\right) \rightarrow \operatorname{End}_{\mathbb{C}}(W)
$$

the corresponding embedding. A routine verification of Definition 3.1 gives the following lemma.

Lemma 6.2. An isomorphism is induced by $j_{\mathfrak{q}}$ from $\tau^{\prime}$ to the representation which is $\mathfrak{q} \times \overline{\mathfrak{q}}$-associated to $\tau$.

The second condition of (23) for $W^{\prime}$ states that

$$
\psi_{W^{\prime}} \in \mathrm{C}^{\omega}\left(G_{\mathbb{C}}^{\prime} / U^{\prime} ; \tau^{\prime}\right) .
$$

Therefore, the above lemma enables us to define the integral $E_{\mathfrak{q}}\left(j_{\mathfrak{q}} \circ \psi_{W^{\prime}}\right)$. Write

$$
E=\frac{\operatorname{deg}(\alpha)}{\operatorname{deg}\left(\alpha^{\prime}\right)} E_{\mathfrak{q}}\left(j_{\mathfrak{q}} \circ \psi_{W^{\prime}}\right) .
$$

An easy calculation shows that the function $E$ satisfies the first condition of (23). Theorem 3.3 states that $E$ satisfies the second condition of (23). It is a consequence of Theorem 4.1 and the $\mathrm{U}(\mathfrak{g})^{K}$-action on $W$ of (18) that $E$ satisfies the third condition of (23). We leave the routine details to the reader. We finally get the main theorem of this paper by Proposition 6.1, as follows.

TheOREM 6.3. We have

$$
\psi_{W}=\frac{\operatorname{deg}(\alpha)}{\operatorname{deg}\left(\alpha^{\prime}\right)} E_{\mathfrak{q}}\left(j_{\mathfrak{q}} \circ \psi_{W^{\prime}}\right)
$$

\subsection{Two examples}

The first example we discuss is the case when $\operatorname{dim}\left(M^{\prime}\right)=1$, i.e. $A_{\mathfrak{q}}(\lambda)$ modules. Let

$$
\lambda: G^{\prime} \rightarrow \mathbb{C}^{\times}
$$

be a continuous character, unitary or not. Still denote by $\lambda$ its holomorphic extension to $G_{\mathbb{C}}^{\prime}$ and its differential. Recall that $A_{\mathfrak{q}}(\lambda)$ is just the cohomologically induced module $\mathcal{R}^{S}(\lambda)$. 


\section{B. SuN}

Fix a maximal torus $T_{\max }$ of $K^{\prime}$ with complexified Lie algebra $\mathfrak{t}$. Then $T_{\max }$ is also a maximal torus of $K$. Let

$$
\Lambda=\left.\lambda\right|_{\mathfrak{t}}+\left.\delta_{\mathfrak{n} \cap \mathfrak{p}}\right|_{\mathfrak{t}}
$$

Assume that $\Lambda$ is dominant with respect to $\mathfrak{n} \cap \mathfrak{k}$, and $\alpha$ is the $K$-type with an extremal weight $\Lambda$. Then $\left.\lambda\right|_{K^{\prime}}$ is the $K^{\prime}$-type $\mathfrak{q}$-associated to $\alpha, \alpha$ is the unique $K$-type which is in the bottom layer of $A_{\mathfrak{q}}(\lambda)$, and $\alpha$ has multiplicity 1 in $A_{\mathfrak{q}}(\lambda)$.

Let $\sigma_{0}$ be an irreducible unitary representation of $K$ on $W_{\alpha}$, of type $\alpha$, as before. Fix a unit vector $v_{0}$ in $W_{\alpha}$, of weight $\Lambda$. Let $P_{\Lambda} \in \operatorname{End}_{\mathbb{C}}\left(W_{\alpha}\right)$ be the orthogonal projection onto $\mathbb{C} v_{0}$. Define a map

$$
\begin{aligned}
\tilde{H}_{\mathfrak{q}}: G & \rightarrow G_{\mathbb{C}}^{\prime}, \\
x & \mapsto y \overline{\theta\left(y^{-1}\right),}
\end{aligned}
$$

where $y \in G_{\mathbb{C}}^{\prime}$ is an element so that $H_{\mathfrak{q}}(x U)=y U^{\prime}$. Fix an identification of $A_{\mathfrak{q}}(\lambda)(\alpha)$ and $W_{\alpha}$ as $K$-modules. Now Theorem 6.3 easily implies

$$
\phi_{A_{\mathfrak{q}}(\lambda), \alpha}\left(k x^{2}\right)=\operatorname{deg}(\alpha) \int_{K}\left(\delta_{\mathfrak{n}} \lambda\right)\left(\tilde{H}_{\mathfrak{q}}(l x)\right) \sigma_{0}\left(k l^{-1}\right) \circ P_{\Lambda} \circ \sigma_{0}(l) d l
$$

for all $k \in K, x \in \exp \left(\mathfrak{p}_{0}\right)$. Write

$$
\tilde{\phi}_{\mathfrak{q}, \lambda}(x)=\frac{1}{\operatorname{deg}(\alpha)} \operatorname{Tr}\left(\phi_{A_{\mathfrak{q}}(\lambda), \alpha}(x)\right), \quad x \in G .
$$

It is a direct consequence of (26) that

$$
\tilde{\phi}_{\mathfrak{q}, \lambda}\left(k x^{2}\right)=\int_{K}\left\langle\sigma_{0}\left(l k l^{-1}\right) v_{0}, v_{0}\right\rangle\left(\delta_{\mathfrak{n}} \lambda\right)\left(\tilde{H}_{\mathfrak{q}}(l x)\right) d l, \quad k \in K, x \in \exp \left(\mathfrak{p}_{0}\right) .
$$

An example of the above formula confirms Li's assumption in [Li97, Theorem 4.3], which enables us to get more information on Gross' conjecture on the discrete spectrum of $\left(G_{2}, P G S p_{6}\right)$.

The second example we will discuss is the lowest weight modules. Assume that $\mathfrak{g}$ is simple, $G$ is of Hermitian type and

$$
\mathfrak{p}=\mathfrak{p}^{+} \oplus \mathfrak{p}^{-},
$$

where both $\mathfrak{p}^{+}$and $\mathfrak{p}^{-}$are $K$ invariant abelian subspaces of $\mathfrak{p}$, and

$$
\mathfrak{p}^{+}=\overline{\mathfrak{p}^{-}} .
$$

Assume

$$
\mathfrak{q}=\mathfrak{k} \oplus \mathfrak{p}^{+} .
$$

Fix an irreducible continuous unitary representation $\sigma_{0}$ of $K$ on $W_{\alpha}$, of type $\alpha$, as before. Define a $(\mathfrak{g}, K)$-module

$$
M=\mathrm{U}(\mathfrak{g}) \otimes_{\mathrm{U}(\overline{\mathfrak{q}})} W_{\alpha},
$$

where we view $W_{\alpha}$ as a $\overline{\mathfrak{q}}$-module via the trivial $\mathfrak{p}^{-}$-action. The $\mathrm{U}(\mathfrak{g})$-action on $M$ is defined via left multiplication, and the $K$-action on $M$ is defined via the tensor product. It is clear that

$$
M(\alpha)=W_{\alpha} .
$$

Note that $M$ is contragredient to a certain cohomologically induced module, with $\alpha$ the unique $K$-type in the bottom layer. By Theorem 6.3, or just check the three conditions of (23), we have

$$
\phi_{M, \alpha}\left(k x^{2}\right)=\int_{K} \sigma_{0}\left(k l^{-1} \tilde{H}_{\mathfrak{q}}(l x) l\right) d l, \quad k \in K, x \in \exp \left(\mathfrak{p}_{0}\right),
$$

where

$$
\tilde{H}_{\mathfrak{q}}: G \rightarrow K_{\mathbb{C}}=G_{\mathbb{C}}^{\prime}
$$

is defined in (25). This formula looks prettier than that given by $\mathrm{Li}$ and Wallach in [Li90]. 


\section{MATRIX COEFFICIENTS}

\section{ACKNOWLEDGEMENTS}

This paper contains the main result of the author's doctoral dissertation at HKUST. Part of the work was also done during the author's stay as a post-doctor at ETH, Zürich. The author would like to thank his thesis advisor, Professor Jian-Shu Li for initiating this project, and for providing help in various forms during and after his stay in Hong Kong. The author would also like to thank Professor Dihua Jiang and the referees for providing many corrections and improvements of the manuscript.

\section{REFERENCES}

Fle80a M. Flensted-Jensen, Discrete series for semisimple symmetric spaces, Ann. of Math. (2) 111 (1980), $253-311$.

Fle80b M. Flensted-Jensen, $K$-finite joint eigenfunctions of $U(\mathfrak{g})^{K}$ on a non-Riemannian semisimple symmetric space $G / H$, Lecture Notes in Mathematics, vol. 880 (Springer, Berlin, 1980), 91-101.

Har58 Harish-Chandra, Spherical functions on a semisimple Lie group, I, Amer. J. Math. 80 (1958), 241-310.

Har75 Harish-Chandra, Harmonic analysis on reductive groups I, J. Funct. Anal. 19 (1975), 104-204.

HL98 M. Harris and J.-S. Li, A Lefschetz property for subvarieties of Shimura varieties, J. Algebraic Geom. 7 (1998), 77-122.

Hoc66 G. Hochschild, Complexification of real analytic groups, Trans. Amer. Math. Soc. 125 (1966), 406-413.

Li90 J.-S. Li, Theta liftings for unitary representations with non-zero cohomology, Duke Math. J. 61 (1990), 913-937.

Li97 J.-S. Li, On the discrete spectrum of $\left(G_{2}, \mathrm{PGSp}_{6}\right)$, Invent. Math. 130 (1997), 189-207.

Kna86 A. Knapp, Representation theory of semisimple groups, an overview based on examples (Princeton University Press, Princeton, NJ, 1986).

KV95 A. Knapp and D. Vogan, Cohomological induction and unitary representations (Princeton University Press, Princeton, NJ, 1995).

SV98 S. Salamanca-Riba and D. Vogan, On the classification of unitary representations of reductive Lie groups, Ann. of Math. (2) 148 (1998), 1067-1133.

Vog79 D. Vogan, The algebraic structure of the representations of semisimple Lie groups I, Ann. of Math. (2) 109 (1979), 1-60.

Vog81 D. Vogan, Representations of real reductive Lie groups (Birkhäuser, Basel, 1981).

Wal92 N. Wallach, Real reductive groups II (Academic Press, San Diego, CA, 1992).

Binyong Sun sun@math.ac.cn

Institute of Mathematics, Academy of Mathematics and System Science, Chinese Academy of Sciences, Beijing 100080, People's Republic of China 